\title{
Now you feel it, now you don't: Motivated attention to emotional content is modulated by age and task demands
}

\author{
Didem Pehlivanoglu ${ }^{1,2} \cdot$ Paul Verhaeghen ${ }^{1}$ \\ Published online: 31 July 2019 \\ (C) The Psychonomic Society, Inc. 2019
}

\begin{abstract}
Several studies with younger adults have examined the degree to which emotion captures attention using the event-related potentials (ERP) technique, but it is unknown whether there are age-related differences on this issue. We examined ERP correlates of age-related differences in processing of task-relevant and task-irrelevant emotional material. Participants viewed emotional or neutral images, presented at fixation, flanked by two bars of either differing or matching orientation. In one set of trials, participants decided whether the pictures were presented in black-and-white or color; in another set of trials, they made a match/judgment on the flanking bars. Before the experiment proper, we determined each individual's threshold for line orientation (in the presence of neutral pictures at fixation); mismatch bar stimuli were constructed using this threshold, thus equating baseline performance on the bar tasks across individuals. When attention was focused on the images, ERPs provided evidence for emotion-based processing in the younger group, regardless of valence; older adults showed more differentiated valence-based processing as reflected by a positivity effect (in line with socioemotional selectivity theory). When the images were taskirrelevant, older adults showed no evidence of emotional processing whatsoever; younger adults showed a pattern of suppression in the form of reduced processing of emotional material relative to neutral images. These findings suggest that, once performance on a neutral baseline task is equated, older adults do not exhibit a specific age-related deficit in inhibiting emotional material; they also suggest qualitative differences in processing of to-be-ignored emotional material in younger and older adults.
\end{abstract}

Keywords Selective attention · Aging; Event-related potentials $\cdot$ Positivity bias $\cdot$ Inhibitory deficit hypothesis $\cdot$ Load theory Socioemotional selectivity theory

There is a growing body of evidence that emotional information may spontaneously capture attention in various tasks, including visual search (Öhman, Flykt, \& Esteves, 2001), spatial orienting (Brosch, Sander, Pourtois, \& Scherer, 2008), or attentional blink (Anderson \& Phelps, 2001), and across various stimulus categories, such as faces, words, or images. Such effects are generally taken to indicate privileged access to awareness for emotionally significant stimuli relative to neutral stimuli when attentional resources are limited in space or time. One approach to study the degree to which emotion captures attention is to ask participants to perform a cognitive task in the context of task-irrelevant, emotional pictures. If the emotional pictures attract attention, as observed,

Didem Pehlivanoglu

dpehlivanoglu@ufl.edu

1 Georgia Institute of Technology, Atlanta, GA, USA

2 Department of Psychology, University of Florida, 945 Center Dr, Gainesville, FL 32603, USA for instance, in a performance decrement in the cognitive task, one could infer that the emotional content is processed even when attention is directed toward another task and location.

Some previous studies that examined the extent to which irrelevant emotional material is processed have employed event-related potentials (ERPs) to assess neural activity associated with emotional processing by specifically focusing on a late-onset positivity component that starts around $300 \mathrm{~ms}$ after stimulus onset at central and central-parietal sites. This ERP component, which also has been referred to as the Late Positive Potential (LPP) (Ito, Larsen, Smith, \& Cacioppo, 1998; Schupp et al., 2000; Weinberg \& Hajcak, 2011), is taken as a neural index of sustained attention to emotional material (Schupp et al., 2000; Schupp, Flaisch, Stockburger, \& Junghöfer, 2006) and is thought to reflect conscious representation of motivationally salient stimuli up to the level of semantic meaning (Hajcak, Dunning, \& Foti, 2009; Schupp et al., 2006). Some studies show that it is larger for emotional compared with neutral content (Cuthbert, Schupp, Bradley, Birbaumer, \& Lang, 2000; Foti, Hajcak, \& Dien, 2009; Weinberg \& Hajcak, 2010). There also is some evidence that 
the late-onset positivity is particularly sensitive to valence, more particularly a bias toward negative emotional content in younger adults (Huang \& Luo, 2006; Ito et al., 1998) and a bias toward positive emotional content in older adults (Kisley, Wood, \& Burrows, 2007; Mathieu et al., 2014).

Several ERP studies with younger adults have examined the degree to which emotion can capture attention by requiring participants to perform a cognitive task in the context of taskirrelevant, emotional pictures. Empirical results from these studies have suggested that ERPs to emotional versus neutral pictures are eliminated when pictures are spatially unattended (De Cesarei, Codispoti, \& Schupp, 2009; Eimer, Holmes, \& McGlone, 2003; Holmes, Kiss, \& Eimer, 2006; Holmes, Vuilleumier, \& Eimer, 2003; MacNamara \& Hajcak, 2009). For example, when each trial showed a vertical pair of faces and a horizontal pair of houses (or vice versa), the late-onset positivity to fearful faces was eliminated when the faces were unattended (Holmes et al., 2003). Also, the late-onset positivity to emotional faces (Eimer et al., 2003) was eliminated when the faces were shown in the periphery and participants performed a cognitive task at fixation. Likewise, the late-onset positivity to emotional images was eliminated if these pictures were shown in the periphery and were unattended (De Cesarei et al., 2009; MacNamara \& Hajcak, 2009). The late-onset positivity was even eliminated when faces were shown at fixation and participants performed a simple one-back task on line bars presented to the left and right of the faces (Holmes et al., 2006). The simplest explanation for these findings can be found in perceptual load theory (Lavie, 1995), which argues that when perceptual load is larger, subjects prioritize task-relevant stimuli, resulting in effective rejection of task-irrelevant distractors. Thus, these findings indicate that ERPs to emotional material might be eliminated when attentional resources are sufficiently depleted by the main task.

Although findings from the ERP studies with younger adults suggest that perception of the background emotional stimuli depends on the task demands associated with the foreground task, it is still an open question whether age-related differences exist in how task demands modulate ERPs for emotional material. Given that we are particularly interested in age-related differences in selective attention for emotional material, it is important to understand how aging is associated with cognitive processes involved in selective attention. The two basic aspects of processing involved in selective attention are inhibition and activation. Inhibition reflects the suppression of the processing of task-irrelevant information, preventing it from gaining access to working memory; activation reflects the enhancement of processing of task-relevant information. According to the inhibitory deficit hypothesis of aging, activation-related processes are mostly preserved in old age, whereas inhibitory functioning declines (Hasher, Stoltzfus, Zacks, \& Rypma, 1991; Hasher \& Zacks, 1988; Lustig, Hasher, \& Zacks, 2007). There is both behavioral and neural evidence for the position that older adults have difficulty in suppressing distractors (Gazzaley et al., 2008; Gazzaley, Cooney, McEvoy, Knight, \& D'esposito, 2005). An important caveat in these studies, however, is that the age-related suppression deficiency in the neural data often disappear once older adults' working memory performance is matched with that of younger adults. Similarly, in her review of the literature, Fabiani (2012) notes that many of the neuroscientific findings about age differences in neural activity associated with inhibition functioning disappear when task difficulty is equated across groups, suggesting that the observed age-related differences in neural data and behavioral performance may not result from the aging process per se but from group differences in mean difficulty levels (see also Verhaeghen, 2011; Verhaeghen, Geigerman, Yang, Montoya, \& Rahnev, 2019).

Thus, in the current study, we investigated ERP correlates of age-related differences in processing of task-relevant and irrelevant emotional material by employing a selective attention task that required younger and older adults to selectively attend to either centrally presented pictures (emotional or nonemotional) or to flanking line bars, concurrently presented on the screen. Our main interest was in comparing age-related differences in the extent to which emotional pictures were still being processed (relative to baseline neutral pictures) at the neural level when they were distractors versus when they were the focus of the task. To circumvent the task difficulty confound mentioned above and equate performance between age groups on a neutral baseline version of the task, we calibrated task difficulty individually (using a similar procedure outlined in Verhaeghen et al., 2019), so that all subjects performed at identical levels in the nonemotional bar task with neutral pictures. The calibration procedure enabled us to examine ERP correlates of age-related differences in inhibiting taskirrelevant emotional material over and beyond task-irrelevant neutral material in the bar task.

The task design additionally allows us to test two competing theories of emotion and aging. According to socioemotional selectivity theory (SST) (Carstensen, Isaacowitz, \& Charles, 1999), motivational priorities change as a function of time left in life. When individuals perceive the future as expansive, they are more likely to focus on information-seeking goals, such as acquiring new information and meeting new people. When individuals perceive the time left as limited, they are more likely to focus on emotionalregulation goals. Given that older adults are more likely to perceive their future as limited, their information processing is claimed to shift toward positive information in order to increase their well-being. Consistent with this theory, many studies show that older adults attend to and remember positive information better than negative information (for a meta-analysis, see Reed, Chan, \& Mikels, 2014), whereas younger adults focus on negative information more than positive 
information (Baumeister, Bratslavsky, Finkenauer, \& Vohs, 2001). In contrast to SST, the aging and brain model (ABM) argues that age-related decline in the brain selectively diminishes emotional arousal in response to negative stimuli (but not positive stimuli), and, as a result, older adults fail to process negative stimuli and thus experience less negative affect (Cacioppo, Berntson, Bechara, Tranel, \& Hawkley, 2011). This perspective then claims that the positivity effect in older adults results from decreased focus on negative material rather than an increased focus on positive material. These two views make opposing arguments about the source of the positivity effect in older age, and so our data, which examine patterns in emotional modulation as a function of task relevancy via ERPs, could be helpful in determining the source of the agerelated differences in emotional processing - either an upregulating of positive content, or a downregulating of negative content.

\section{Expected results}

Picture task In the picture task, emotional modulation would change based on ERP components and age. The literature suggests that the late-onset positivity component would be sensitive to motivationally salient stimuli (Carretié et al., 2001; Ito et al., 1998; Kisley et al., 2007). Thus, we expect that this component would capture both the positivity bias in older adults and the negativity bias in younger adults.

Regarding the source of the positivity bias in older adults, if the age-related positivity bias results from an increased focus on positive material as suggested by SST (Carstensen et al., 1999), then older adults would show larger ERPs for positive pictures compared with neutral and negative images relative to younger adults. If, however, the positivity bias is driven by a reduced negativity bias in older adults as suggested by ABM (Cacioppo et al., 2011), then older adults would show reduced ERPs for negative images compared with positive and neutral images compared with younger adults, with no age-related differences in the ERP amplitudes between positive and neutral images.

Bar task In the bar task, the goal was to investigate age-related differences in emotional modulation of ERPs when pictures are unattended. Under the hypothesis that the often-reported, age-related deficit in inhibiting task-irrelevant emotional material is an artifact of differences between age groups at baseline, we would expect no age-related effects in ERPs for taskirrelevant material at all, given that the calibration procedure sets the baseline performance levels to be identical across age groups. Thus, if there is no age-related deficit in inhibiting task-irrelevant emotional, ERPs for task-irrelevant emotional and neutral material would be similar within and between age groups. If, however, there is an age-related deficit in inhibiting task-irrelevant emotional material, the bar task should yield age-related differences in the emotional ERP components even after performance is equated, because the task demand associated with the bar task may prevent older adults from exerting suppression of task-irrelevant emotional material. That is, older adults would show larger ERPs for taskirrelevant emotional material relative to neutral material during the bar task, whereas ERPs for emotional and neutral pictures would be similar for younger adults.

\section{Methods}

\section{Participants}

Thirty-four younger and 35 older adults participated in the experiment. All participants provided written, informed consent before participating in this study according to a protocol approved by the Georgia Institute of Technology's Human Subjects Institutional Review Board and in accordance with the principles expressed in the Declaration of Helsinki. Older participants were recruited from the community; they received cash payment (\$10/hour) as compensation for participation. ${ }^{1}$ Younger adults were students at the Georgia Institute of Technology and participated in the study in return for course credit. Data from two younger and three older participants were excluded from further analysis due to slow response times (response times 2 standard deviations above the group mean) and/or more than 50\% trials with ERP artifacts (e.g., alpha, eye movements, blinks, muscle tension). The mean age of the remaining 32 younger adults (16 females) was 19.97 $(S D=1.58)$; mean age of the remaining 32 older adults (18 females) was $70.59(S D=4.1)$. All participants were righthanded, with normal or corrected to normal vision, with no reports of psychiatric or neurological disorders, vascular disease, or color blindness. None of the participants were taking CNS-active medications. None of the participants reported that they were suffering from depression or anxiety. Sixtysix percent of the older adults reported being White/ Caucasian and 34\% Black/African-American; $44 \%$ of the young adults reported being White/Caucasian, $9 \%$ of Hispanic descent, 9\% reported being of Black/AfricanAmerican descent, 32\% reported being of Asian descent, and $6 \%$ reported being of other descent. Older adults had completed more years of education $(M=16.97, S D=2.96)$ than younger adults $(M=13.83, S D=1.6)[t(62)=5.29, p<$ $0.001]$. Younger adults $(M=60.69, S D=11.5)$ performed significantly better than older adults $(M=46.66, S D=7.16)$ on a symbol-digit test (Smith, 1973) $[t(62)=5.86, p<0.001]$.

\footnotetext{
${ }^{1}$ We could not perform an a-priori power analysis to determine the sample size, because there were no previous aging studies on this topic. Given that the expected effect size is unknown, we included a number of participants comparable to that of previous similar studies.
} 
Table 1. Mean valence, minimum, and maximum ratings for the stimuli

\begin{tabular}{|c|c|c|c|c|}
\hline Image type & Younger & Min - Max & Older & Min - Max \\
\hline Negative & $7.15(0.64)$ & $5.76-8.61$ & $6.88(0.50)$ & $5.83-7.92$ \\
\hline Positive & $2.65(0.55)$ & $1.38-4.14$ & $3.43(0.47)$ & $2.10-4.24$ \\
\hline Neutral (Actual Exp.) & $5.03(0.21)$ & $4.57-5.48$ & $5.03(0.20)$ & $4.50-5.50$ \\
\hline Neutral (Calibration Exp.) & $5.03(0.27)$ & $4.52-5.43$ & $5.03(0.23)$ & $4.52-5.43$ \\
\hline
\end{tabular}

Standard deviations in parentheses. Min refers to minimum and max refers to maximum.

Older adults' performance $(M=36.28, S D=2.8)$ on the Shipley Vocabulary test (Shipley, 1946) was significantly higher than performance of younger adults $(M=31.06, S D$ $=3.17)[t(62)=6.98, p<0.001]$.

\section{Stimuli}

Given that older adults, relative to younger adults, tend to rate emotional pictures as more extreme for both valence and arousal (Grühn \& Scheibe, 2008), it is important to select stimuli for which subjective ratings are matched between age groups. To this end, we conducted a picture rating study prior to the experiment proper. In this rating experiment, independent groups of younger and older adults rated valence and arousal level of emotional stimuli. For the picture rating experiment, a total of 720 images, divided evenly between valence categories (neutral, negative, positive) as judged by the experimenters, were selected from the International Affective Picture Set (IAPS) (Lang, Bradley, \& Cuthbert, 1999), the Nencki Affective Picture System (NAPS) (Marchewka, Żurawski, Jednoróg, \& Grabowska, 2014), the Open Affective Standardized Image Set (OASIS) (Kurdi, Lozano, \& Banaji, 2017), the Geneva affective picture database (GAPED) (Dan-Glauser \& Scherer, 2011), and Google (using search terms, such as celebration, mutilation, etc.). Because previous research showed that there are age-related differences in ratings of pictures with radical/exciting sports and erotic content (Backs, da Silva, \& Han, 2005), pictures from these semantic categories were excluded. Additionally, an effort was made to select images with similar visual complexity (indexed by jpeg size; Donderi, 2006; Marchewka et al., 2014), because a previous study by Wiens, Sand, and Olofsson (2011b) showed that picture composition (i.e., figure vs. scene) confounds ERP amplitudes.

Participants were recruited through Amazon's Mechanical Turk (MTurk) and completed the study online. Participants rated each presented picture in terms of valence (on a 1-9 point scale, where 1 was extremely pleasant and 9 was extremely unpleasant) and arousal (on a 1-9 point scale, where 1 was extremely aroused and 9 was extremely calm). Procedural details can be found in the Appendix. We selected a total of 462 pictures that had been rated as positive and arousing $(\mathrm{N}=$ 140; valence less than 4.25 and arousal less than 6.4), negative and arousing $(\mathrm{N}=140$; valence greater than 5.75 , arousal less than 6), and neutral and non-arousing ( $\mathrm{N}=182$; valence between 4.5 and 5.5, arousal greater than 6). Of the 182 neutral pictures, 42 were used in the calibration experiment that participants performed prior to the experiment proper. Complexity of pictures selected for the experiment (indexed by JPEG size) did not differ significantly between valence categories. The mean valence and arousal ratings for each picture category and age group for the stimuli included in the study are presented in Tables 1 and 2 .

\section{Procedure}

Before the session, each participant signed a consent form, filled out the Shipley Vocabulary test and symbol-digit test measuring verbal ability and processing speed, respectively. Next, they completed the calibration experiment which was followed by the actual experiment. EEG was only recorded during the actual experiment. In both experiments, the subjects sat approximately $57 \mathrm{~cm}$ from the display. Both experiments were run on a 17-inch CRT monitor (1024 x 768 pixel resolution; $75 \mathrm{~Hz}$ refresh rate) using MATLAB (The MathWorks, Natick, MA) and the Psychophysics Toolbox (Brainard, 1997; Pelli, 1997).

Calibration experiment Trial structure for the calibration experiment is presented in Fig. 1. Each trial started with the presentation of a fixation cross for $1,500 \mathrm{~ms}$. Next, a centrally presented neutral picture $\left(6.5^{\circ} \times 5.5^{\circ}\right)$ and two bars $\left(0.3^{\circ} \mathrm{x}\right.$ $\left.2.75^{\circ}\right)$ positioned peripherally $\left(2^{\circ}\right.$ to the right and left of the central picture) were presented briefly. Presentation duration of the stimuli was $200 \mathrm{~ms}$ for the younger participants and $250 \mathrm{~ms}$ for the older participants, below the time needed to execute a saccade. Presentation duration for older adults was increased to allow for extra processing time due to age-related slowing; the slowing factor was derived from the typical agerelated slowing factor for saccadic eye movements (Verhaeghen, 2013). Only neutral pictures were used for the calibration experiment (see Padmala \& Pessoa, 2014, for a similar calibration procedure). Following the stimulus presentation, the subjects were asked to withhold their response for $800 \mathrm{~ms}$, during which period a blank screen was presented (this mimics the procedure in the actual experiment). Next, 
Table 2. Mean arousal, minimum, and maximum ratings for stimuli

\begin{tabular}{|c|c|c|c|c|}
\hline Image type & Younger & Min - Max & Older & Min - Max \\
\hline Negative & $4.50(0.69)$ & $3.33-5.96$ & $4.50(0.61)$ & $2.95-5.86$ \\
\hline Positive & $5.55(0.82)$ & $3.74-6.60$ & $5.53(0.59)$ & $4.00-6.38$ \\
\hline Neutral (Actual Exp.) & $6.97(0.54)$ & $6.00-8.44$ & $6.97(0.38)$ & $6.04-7.77$ \\
\hline Neutral (Calibration Exp.) & $6.97(0.60)$ & $4.42-5.43$ & $6.98(0.41)$ & $4.52-5.43$ \\
\hline
\end{tabular}

Standard deviations in parentheses. Min refers to minimum and max refers to maximum.

the subjects were shown a question mark on the center of the screen and asked to indicate whether the orientations of the peripheral bars matched.

We used the QUEST algorithm (Watson \& Pelli, 1983), an adaptive staircase procedure, to determine the $75 \%$ discrimination threshold for the angular difference of the bars for each subject. The number of trials needed to estimate the $75 \%$ threshold was determined by the algorithm and the experiment stopped when the performance criterion was achieved for each participant. ${ }^{2}$ The calibration experiment took approximately $10 \mathrm{~min}$ to complete.

Actual experiment Following the calibration experiment, EEG capping was done for the actual experiment. The trial structure in the actual experiment (Fig. 2) was the same as the calibration experiment except that each trial started with the presentation of a small circle for $1,500 \mathrm{~ms}$ to allow the subjects to blink (if necessary) and thus to avoid eye blink artifacts during stimulus presentation. The individual threshold obtained from the calibration experiment was used to set differences in angle of bar rotation on an individual basis. There were two tasks: In the bar task, subjects were instructed to ignore the central images and to indicate whether the orientations of the peripheral bars matched; they did this while maintaining fixation on the center of the screen, where the picture appeared. In the picture task, subjects were instructed to ignore the bars and to indicate whether the picture was presented in black and white. ${ }^{3}$ Likewise, they maintained fixation at the center of the screen, where the picture appeared.

Subjects performed the bar and picture tasks in separate blocks; task order was counterbalanced across participants. The picture and bar task in the current study were adopted from previous studies employing similar tasks (see Sand \&

\footnotetext{
2 The minimum number of trials to achieve the performance criterion was set at 40 ; the maximum number at 100 . The average number of trials needed to reach the performance criterion was 74.1 trials for younger adults and 75.6 trials for older adults.

${ }^{3}$ One potential concern could be that using grayscale emotional pictures may diminish affective modulation associated with picture perception, especially considering the fact that the stimuli will be presented very briefly in the study. Codispoti, De Cesarei, and Ferrari (2012) tested this possibility and they found that affective modulation does not depend on picture color; the ERPs were larger for emotional compared to neutral regardless of whether the pictures were colored or in grayscale.
}

Wiens, 2011; Wiens, Sand, Norberg, \& Andersson, 2011a, for the picture task; see Erthal et al., 2005; Padmala \& Pessoa, 2014; Pessoa, Kastner, \& Ungerleider, 2002; Verhaeghen et al., 2019, for the bar task). Each task consisted of 5 blocks of 42 trials; each block contained an equal number of neutral, positive, and negative images, an equal number of color and black and white images, and an equal number of match and mismatch stimuli. The order of neutral and emotional images, color and black and white images, and match and mismatch bars within a block was randomized. Additionally, picture color was counterbalanced across participants, such that pictures that were presented in black and white for one half of the participants were presented in color for the other half, and vice versa. For mismatch bar stimuli, the angular difference between the two bars was created by rotating one of the bars either clockwise or counter clockwise one threshold value away from the original stimulus. The subjects were given feedback about accuracy at the end of each block. The whole session (calibration and experiment proper) lasted approximately $100 \mathrm{~min}$.

\section{EEG Acquisition}

Scalp-recorded EEG data was collected from $32 \mathrm{Ag}-\mathrm{Ag} / \mathrm{Cl}$ electrodes using an ActiveTwo amplifier system (Biosemi, Amsterdam, Netherlands). Electrodes were positioned according to the extended 10-20 system (Nuwer et al., 1998). Electrodes were located at left/right hemisphere locations (FP1/FP2, AF3/AF4, F3/F4, F7/F8, FC1/FC2, FC5/FC6, $\mathrm{C} 3 / \mathrm{C} 4, \mathrm{~T} 7 / \mathrm{T} 8, \mathrm{CP} 1 / \mathrm{CP} 2, \mathrm{CP} 5 / \mathrm{CP} 6, \mathrm{P} 3 / \mathrm{P} 4, \mathrm{P} 7 / \mathrm{P} 8, \mathrm{PO} 3 /$ $\mathrm{PO} 4, \mathrm{O} 1 / \mathrm{O} 2)$ as well as midline sites $(\mathrm{Fz}, \mathrm{Cz}, \mathrm{Pz}, \mathrm{Oz})$. Two additional electrodes placed on the left and right mastoid processes were used as off-line references. Four additional electrodes were placed above and below the left eye to record a vertical electrooculogram (VEOG) and on the outer canthi of the left and right eyes to record a horizontal electrooculogram (HEOG). EEG was recorded with 24-bit resolution and a sampling rate of $512 \mathrm{~Hz}$. All data processing, including filtering and extracting epochs, was performed in MATLAB using the ERPLAB (Lopez-Calderon \& Luck, 2014) toolbox, except for artifact correction and rejection procedures which were performed using the EEGLAB toolbox (Delorme \& Makeig, 2004). For data processing, electrodes placed on the mastoids 
were used as off-line references and data were digitally bandpass filtered using a 2nd order infinite impulse response (IIR) Butterworth filter (half-amplitude cutoffs at 0.01 and $100 \mathrm{~Hz}$, $12 \mathrm{~dB} /$ octave roll-off). Then, EEG segments were created from 200-ms prestimulus onset until 1,000-ms poststimulus onset. Artifacts were removed in 3 steps. First, manual artifact rejection procedure was applied to remove epochs containing non-ocular artifacts (e.g., large drift, electrode spikes, saturation). Second, independent component analysis was used to remove ocular artifacts components from the remaining epochs (Delorme \& Makeig, 2004). Lastly, epochs containing uncorrected artifacts $( \pm 150 \mathrm{mV})$ were removed. Depending on the condition and age group, between $7.6 \%$ and $14 \%$ of trials were removed (median $=9.4 \%$ ). Epochs were averaged separately for each participant, valence, electrode, and task. The averaged waveforms were digitally smoothed with a low-pass filter of $30 \mathrm{~Hz}$. Only correct trials (i.e., hits and correct rejections) were included in the ERP analysis.

\section{ERP Analysis}

Based on the extant ERP studies reporting late-onset positivity in similar experimental designs (Holmes et al., 2006; Sand \& Wiens, 2011), the late-onset positivity was examined at central and centro-parietal electrode sites (C3, C4, CP1, CP2).

To detect time windows where younger and older adults showed reliable emotional processing in the picture and bar tasks, ERPs elicited by the emotional material versus neutral images at electrode sites of interest in each task and within each age group were submitted to two-tailed cluster-based permutation tests with a family-wise alpha level of 0.05 (Bullmore et al., 1999) using the Mass Univariate ERP Toolbox (Groppe, Urbach, \& Kutas, 2011). These clusterbased multiple comparisons corrections provide control over the weak family-wise error rate while maintaining high sensitivity to detect broadly distributed ERP effects (Groppe et al., 2011; Maris \& Oostenveld, 2007). The ERP data were downsampled to $128 \mathrm{~Hz}$ before these mass univariate analyses. All time points (measured every $4 \mathrm{~ms} ; 90$ total time points) between 100 and $800 \mathrm{~ms}$ at electrode sites of interest (C3, C4, CP1, CP2) were included in the analyses (i.e., 360 total comparisons). To estimate the distribution of the null hypothesis, 2,500 random within-participant permutations of the data were applied, which is more than twice the number recommended for a family-wise alpha level of 0.05 (Manly, 1997). For each permutation, all $t$-scores corresponding to uncorrected $p$-values of 0.05 or less were formed into clusters with any neighboring t-scores meeting the same criteria. Electrodes within $5 \mathrm{~cm}$ of one another were considered spatial neighbors and adjacent time points were considered temporal neighbors. The cluster "mass" was defined as the sum of the $t$-scores in each cluster, and the most extreme cluster mass in each of the 2,501 sets of tests was recorded and used to estimate the distribution of the null hypothesis. The permutation cluster mass percentile ranking of each cluster from the observed data was used to derive its $p$-value. The $p$-value of the cluster was assigned to each member of the cluster and $t$-scores that were not included in a cluster were assigned a $p$-value of 1 . Once time windows of interest for ERPs reflecting emotion processing were determined via the cluster-based permutation analyses for each age group within the each task, a mixed ANOVA including factors of Task, Valence, and Group was performed for further statistical testing of ERP effects across tasks and age groups. ${ }^{4}$

\section{Results}

\section{Calibration Experiment Results}

Bar task performance of younger adults $(M=0.75, S D=0.04)$ in the calibration experiment was similar to that of the older adults $(M=0.76, S D=0.06)[t(62)=0.33, p=0.75]$, also indicating that the desired level of accuracy $(75 \%)$ was indeed achieved. Younger adults $\left(M=10.34^{\circ}, S D=4.11^{\circ}\right)$ had lower thresholds for angular disparity than older adults $\left(M=15.3^{\circ}\right.$, $\left.S D=6.79^{\circ}\right),[t(62)=3.53, p=0.001]$.

\section{Actual Experiment: Behavioral Results}

A Group (Younger, Older) x Task (Bar, Picture) x Valence (Positive, Negative, Neutral) ANOVA was conducted on correct responses and corresponding reaction times. Proportions of correct and corresponding response times for negative, positive, and neutral valences in each task are presented in Table 3. With regard to accuracy, the ANOVA revealed a main effect of Task $\left[F(1,62)=294.11, p<0.001, \eta_{\mathrm{p}}^{2}=0.83\right]$ and a significant Task $\mathrm{x}$ Valence interaction $[F(2,124)=5.10, p=$ $\left.0.007, \eta_{\mathrm{p}}^{2}=0.08\right]$. The Task $\mathrm{x}$ Valence interaction reflects that accuracy for both negative and positive images was larger than accuracy for neutral images in the picture task [all $t \mathrm{~s}>$ $1.91, p s<0.001]$, while there was no effect of valence on accuracy in the bar task [all $t \mathrm{~s}<0.64, p \mathrm{~s}>0.5]^{5}$ Additionally, accuracy was higher in the picture task than in the bar task regardless of image valence [all $t \mathrm{~s}>13.76, p \mathrm{~s}<$

\footnotetext{
${ }^{4}$ We did not include hemisphere as a factor in the ANOVA analyses, because (i) there is no reason to expect hemispheric differences, and (ii) no hemisphere by valence and/or task interactions were in fact obtained. Likewise, given a lack of a-priori hypotheses about the relevant topographies associated with the ERP components of interest, location was not included as a factor in the analyses.

${ }^{5}$ We also calculated corrected recognition rates (proportion of hit rates minus false alarm rates) in the bar task for each participant. A Group (Young, Older) $\mathrm{x}$ Valence (Positive, Negative, Neutral) ANOVA on corrected recognition rates in the bar task revealed no significant main effects or interactions [all $F_{\mathrm{S}}<$ $0.58, p s>0.26]$. Calculating corrected recognition rates for the picture task was not possible as there is no way to assess false-alarm rates.
} 


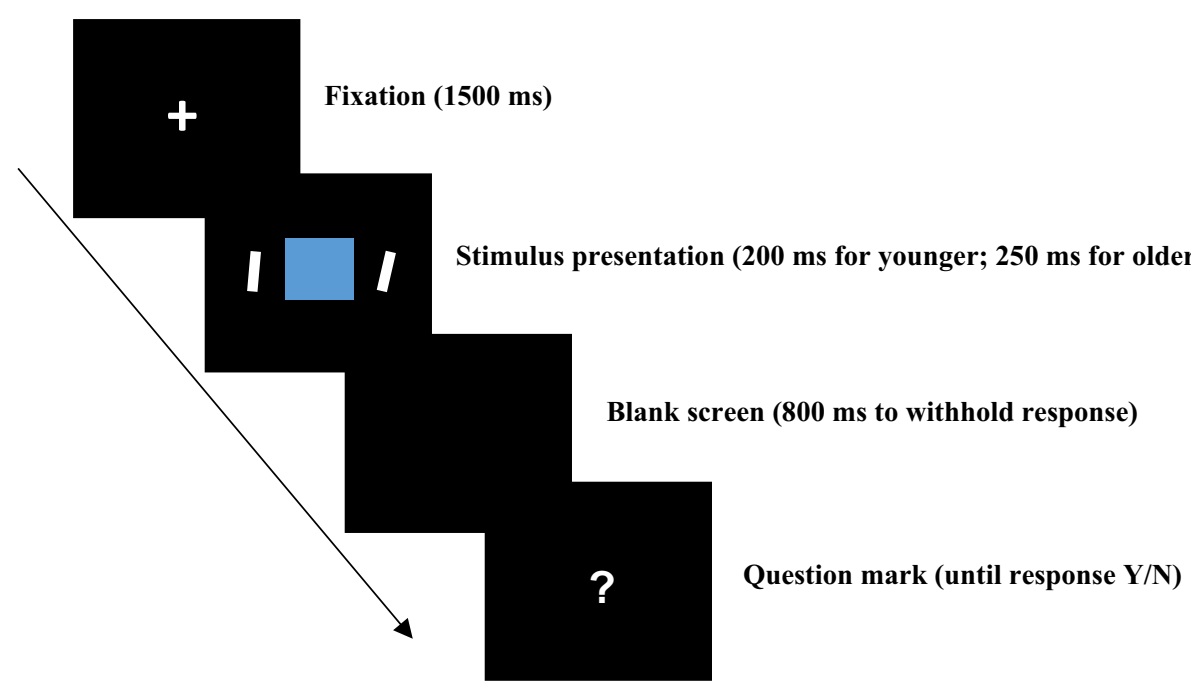

Fig. 1. Trial structure for the calibration experiment: Each trial started with presentation of a fixation cross for $1,500 \mathrm{~ms}$. After that, a neutral picture (represented by a blue square) flanked by two bars was shown briefly. This was followed by presentation of a blank screen for $800 \mathrm{~ms}$

0.001]. Note that the picture task clearly shows a ceiling effect, potentially masking true emotion effects. Neither a main effect of valence nor interaction effects involving age group was significant (all $F \mathrm{~s}<2.6, p \mathrm{~s}>0.1$ ). With regard to response times, the ANOVA revealed a main effect of Group $[F(1,62)=$ 6.71, $p=0.01, \eta_{\mathrm{p}}^{2}=0.9$ ], indicating that older adults responded slower than younger adults. Neither main effects of Task and Valence nor other interaction effects involving Age Group, Task, and/or Valence factors was significant [all $\left.F_{\mathrm{S}}<0.7, p \mathrm{~s}>0.1\right]^{6}$

\section{Actual Experiment: ERP Results}

The cluster-based permutation test Raster diagrams visualizing the results of cluster-based permutation analyses for a contrast comparing ERPs to emotional and neutral images, and the corresponding topographic maps within each age group are presented in Fig. 3 for the picture task, and in Fig. 4 for the bar task. In the picture task, one significant positive cluster $(p<0.05)$ lasting from 564 to $775 \mathrm{~ms}$ was identified in the younger group and one significant positive cluster $(p<$ 0.05 ) lasting from 463 to $650 \mathrm{~ms}$ was identified in the older group. In the bar task, one significant negative cluster $(p<$ 0.05 ) lasting from 252 to $627 \mathrm{~ms}$ was identified in the younger group, whereas no significant positive or negative clusters ( $p$

\footnotetext{
${ }^{6}$ We also tested for interference from emotional material in the bar task by analyzing the data only from incorrect responses from the bar task. A set of Group (Young, Older) x Valence (Positive, Negative, Neutral) ANOVAs on overall proportion of incorrect and false alarm rates in the bar task showed no effect of Valence, nor any Valence $\mathrm{x}$ Group interactions (all $F \mathrm{~s}<0.37, p \mathrm{~s}>$ 0.7). Similarly, another set of analyses on response times for overall proportion of incorrect responses and false alarms did not yield any significant main effects of Valence, nor any Valence x Group interactions (all $F$ s $<1.25$, $p$ s $>$ $0.29)$
}

during which the subjects were asked to withholder their responses. Immediately after, the subjects were shown a question mark and decided as quickly and accurately as possible whether or not the orientations of the bars matched.

$>0.24)$ were identified in the older group. Thus, based on the cluster-based permutation analyses, ERP effects were defined as the mean amplitude from 463 to $650 \mathrm{~ms}$ time window in both tasks for the older group. For the younger group, ERP effects were defined as the mean amplitude from 564 to $775 \mathrm{~ms}$ time window in the picture task and from 252 to $627 \mathrm{~ms}$ time window in the bar task.

ERP effects A Task x Valence x Group ANOVA revealed a main effect of Group $\left[F(1,62)=28.24, p<.001, \eta^{2}=0.32\right]$ that was modified by interactions with Task $[F(1,62)=37.58$, $\left.p<0.001, \eta_{\mathrm{p}}^{2}=0.38\right]$, and Valence $[F(2,124)=4.56, p=0.01$, $\left.\eta_{\mathrm{p}}^{2}=0.07\right]$. An interaction between Valence and Task was also significant $\left[F(2,124)=30.57, p<0.001, \eta^{2}=0.33\right]$. Importantly, there was a Task $\mathrm{x}$ Valence $\mathrm{x}$ Group interaction $\left[F(2,124)=5.49, p=0.005, \eta_{\mathrm{p}}^{2}=0.08\right]$. To follow-up this interaction, we conducted analyses within the picture and bar task separately in two steps. First, we examined the effect of Valence within-age group. In the second step, we examined between-age group differences on ERP difference scores reflecting positivity (positive minus neutral), negativity (negative minus neutral), and emotional salience (average emotional minus neutral) effects at the electrode sites of interest in the time windows associated with ERP effects in each age group.

Picture task Figure 5 displays the average ERP waveforms elicited by negative, neutral, and positive images obtained from correct trials in the picture task for younger and older adults.

Younger adults The ANOVA during the $564-775 \mathrm{~ms}$ time window revealed a main effect of Valence $[F(2,62)=$ 


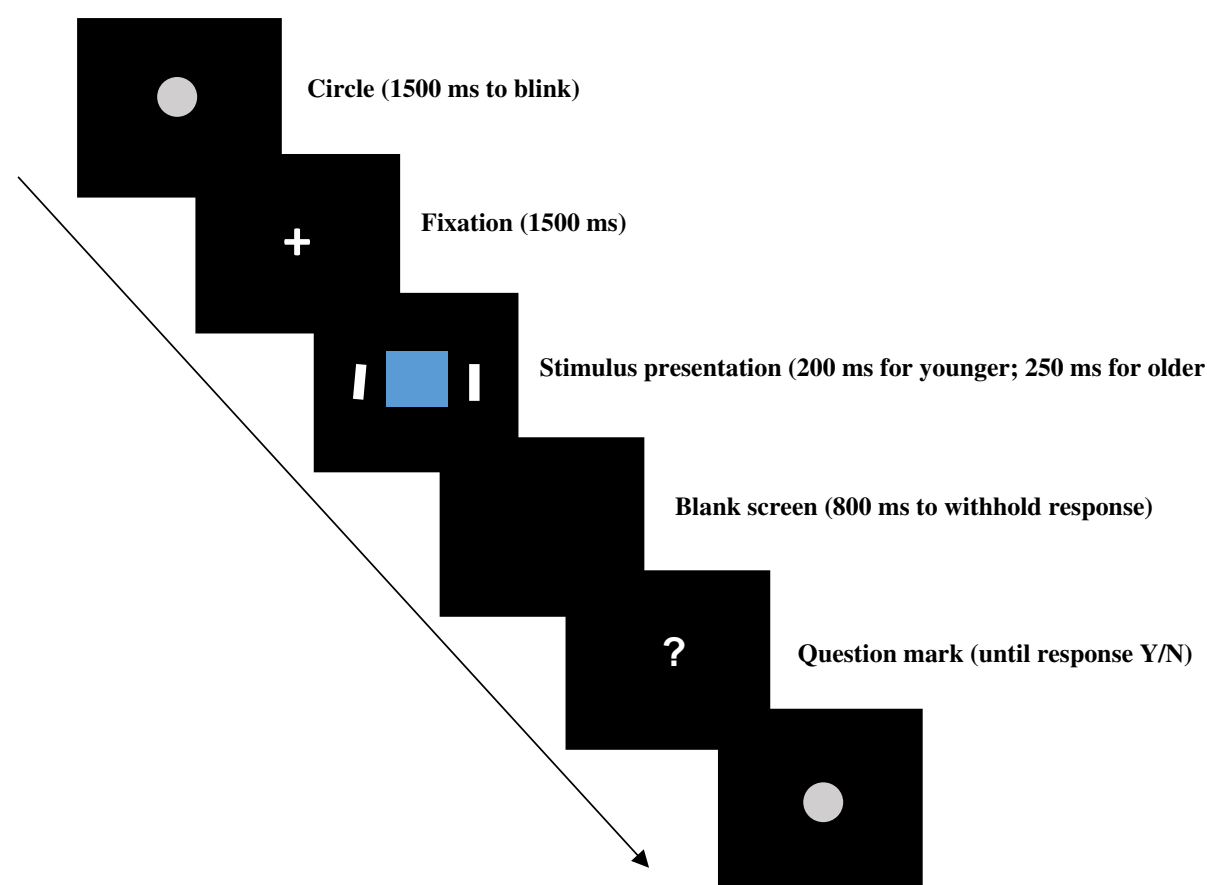

Fig. 2. Trial structure for the actual experiment: Each trial started with presentation of a circle for $1,500 \mathrm{~ms}$ to signal to the subjects to blink. Then, a fixation cross was shown for $1,500 \mathrm{~ms}$. After that, a neutral, negative, or positive picture (represented here by a blue square) flanked by two bars was shown briefly. This was followed by presentation of a

7.41, $\left.p=0.001, \eta_{p}^{2}=0.19\right]$. As can be seen in Fig. 5, follow-up analyses (using a Bonferroni corrected $\alpha=$ 0.0167) showed that ERPs were larger for both positive $(M=6.43 \mu \mathrm{V}, S D=8.04)$ and negative $(M=5.8 \mu \mathrm{V}, S D$ $=6.68)$ images than neutral $(\mathrm{M}=3.15 \mu \mathrm{V}, \mathrm{SD}=7.39)$ images in the picture task [all $t s>3.25, p s<0.004$ ] with no significant difference between negative and positive images $[t(31)=0.58, p=0.56]$.

Older adults The ANOVA during the 463-650 ms time window revealed a main effect of Valence $[F(2,62)=$ 12.49, $\left.p<0.001, \eta_{\mathrm{p}}^{2}=0.29\right]$. As shown in Fig. 5,

Table 3. Proportion correct and corresponding reactions times in the picture and bar task for younger and older adults

\begin{tabular}{|c|c|c|c|c|}
\hline & Younger & & Older & \\
\hline \multicolumn{5}{|l|}{ Picture Task } \\
\hline Negative & $.98(.01)$ & 455 (116) & $.97(.04)$ & $540(207)$ \\
\hline Neutral & $.97(.02)$ & 452 (118) & $.95(.06)$ & $525(179)$ \\
\hline Positive & $.99(.02)$ & 434 (110) & $.98(.02)$ & $514(157)$ \\
\hline \multicolumn{5}{|l|}{ Bar task } \\
\hline Negative & $.72(.09)$ & 448 (162) & $.75(.13)$ & $516(142)$ \\
\hline Neutral & $.72(.10)$ & 428 (189) & $.76(.12)$ & $514(164)$ \\
\hline Positive & $.73(.10)$ & $428(136)$ & $.75(.12)$ & $553(155)$ \\
\hline
\end{tabular}

Standard deviations in parentheses. blank screen for $800 \mathrm{~ms}$ during which the subjects were asked to withholder their responses. Immediately after, the subjects were shown a question mark and decided as quickly and accurately as possible as whether or not the orientations of the bars matched (bar task) or whether the picture was presented in black and white (picture task)

follow-up analyses (using a Bonferroni-corrected $\alpha=$ 0.0167) showed that ERPs were larger for positive $(M$ $=10.71 \mu \mathrm{V}, S D=7.1)$ than negative $(M=8.74 \mu \mathrm{V}, S D$ $=7.2)$ and neutral $(M=7.08 \mu \mathrm{V}, S D=6.59)$ images in the picture task [all $t s>2.8, p s<0.001$ ] with no difference between negative and neutral images $[t(31)$ $=2.34, p=0.02]$.

Between-group analyses Figure 6 displays difference waveforms reflecting positivity (positive minus neutral), negativity (negative minus neutral), and emotional salience (average emotional minus neutral) effects in the picture task. During the window associated with the late-onset positivity component in each age group, the analyses revealed that the magnitude of emotion related ERP effects was similar across age groups [all $t s<1, p s>0.1$ ].

Bar task Figure 7 displays the average ERP waveforms elicited by negative, neutral, and positive images obtained from correct trials in the bar task for younger and older adults.

Younger adults As shown in Fig. 7, visual inspection of ERP waveforms suggested that the late-onset positivity in younger adults was preceded by a non-hypothesized sustained negative deflection. Therefore, we conducted two ANOVAs to investigate these qualitatively different ERP effects separately. Regarding the negative deflection, 


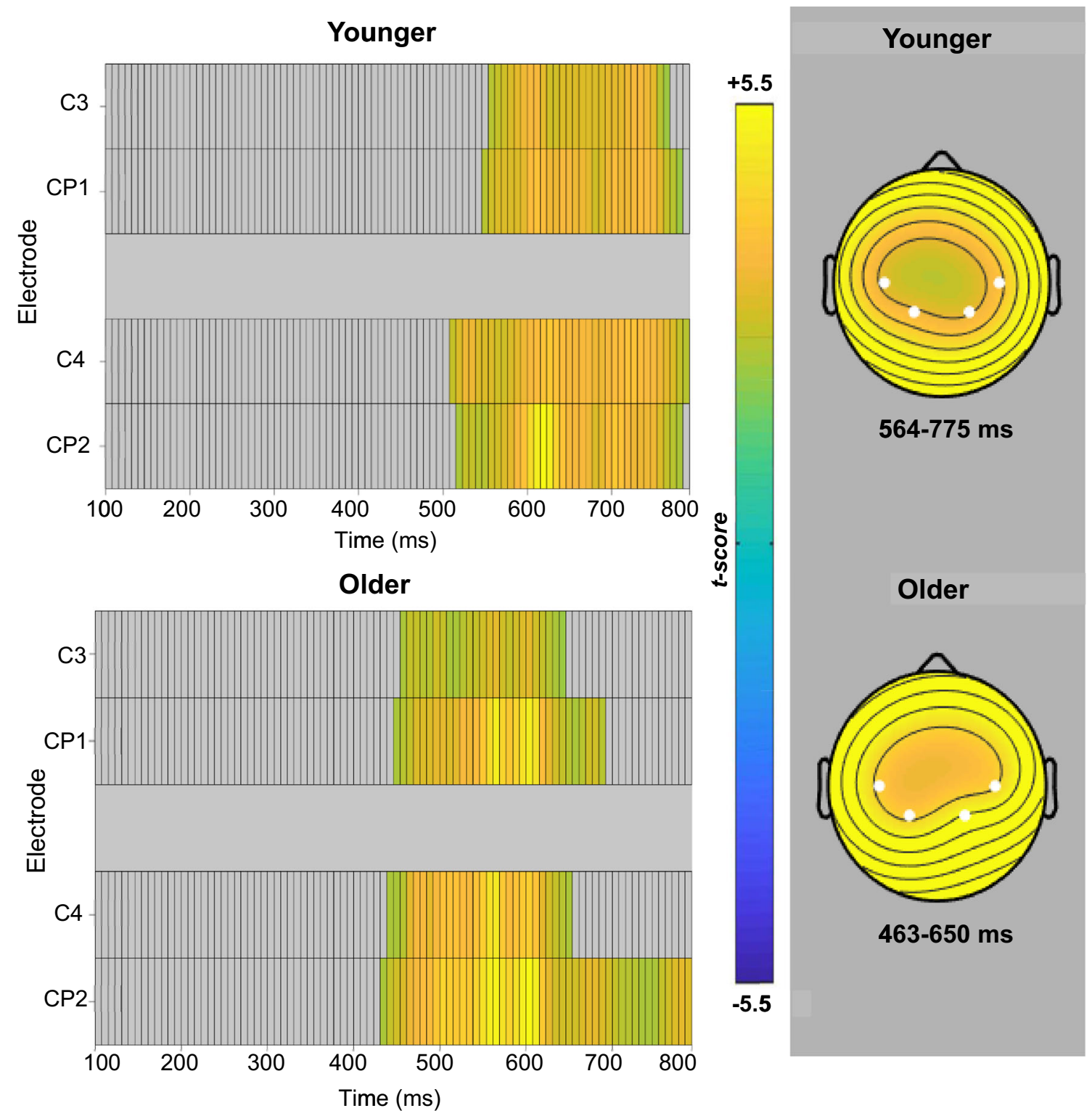

Fig. 3. Picture task results. Left-hand panel: Raster diagrams visualizing the results of cluster-based permutation analyses on ERPs for the contrast comparing emotional and neutral images. Each cell represents the result of a $t$-test on the contrast. The $\mathrm{y}$-axis indicates electrodes. The $\mathrm{x}$-axis indicates time in $13-\mathrm{ms}$ increments. Color indicates that the test was

the ANOVA during the 252-400 ms time window revealed a main effect of Valence $\left[F(2,62)=19.57, p<0.001, \eta_{p}^{2}\right.$ $=0.39$ ]. Follow-up analyses (using a Bonferroni-corrected $\alpha=0.0167)$ showed that ERPs were larger for negative $(M=-8.17, S D=9.68)$ and positive $(M=-10.35 \mu \mathrm{V}, S D$ $=9.89)$ images than neutral $(M=-4.37 \mu \mathrm{V}, S D=11.63)$ images [all $t s>3.5$, ps $<0.01$ ] with no significant difference between negative and positive images $[t(31)=2.57$, $p=0.02]$. Regarding the late-onset positivity, the ANOVA during the 401-627 ms time window revealed a main effect of Valence $\left[F(2,62)=10.24, p<0.001, \eta_{p}^{2}=0.25\right]$. Follow-up analyses (using a Bonferroni corrected $\alpha=$ significant $(p<0.05)$ after correcting for multiple comparisons. Righthand panel: Topographical maps reflecting difference scores for ERPs to emotional versus neutral images. Circles represent electrode locations $(\mathrm{C} 3 / \mathrm{C} 4, \mathrm{CP} 1 / \mathrm{CP} 2)$ for the cluster analysis

0.0167) showed that ERPs were larger for neutral $(M=$ $7.02, S D=10.54)$ than positive $(M=1.85 \mu \mathrm{V}, S D=8.47)$ and negative $(M=2.74 \mu \mathrm{V}, S D=9.77)$ images [all $t s>3$, $p s<0.01]$ with no significant difference between negative and positive images $[t(31)=0.8, p=0.43]$.

Additionally, accuracy in the bar task was positively correlated with difference scores denoting positivity $(\mathrm{r}=$ $0.58, p=0.001)$, negativity $(r=0.36, p=0.04)$, and emotional salience ( $r=0.51, p=0.003$ ) during the time window associated with late-onset positivity, suggesting that suppression of emotional material in the bar task increases accuracy in the bar task. 


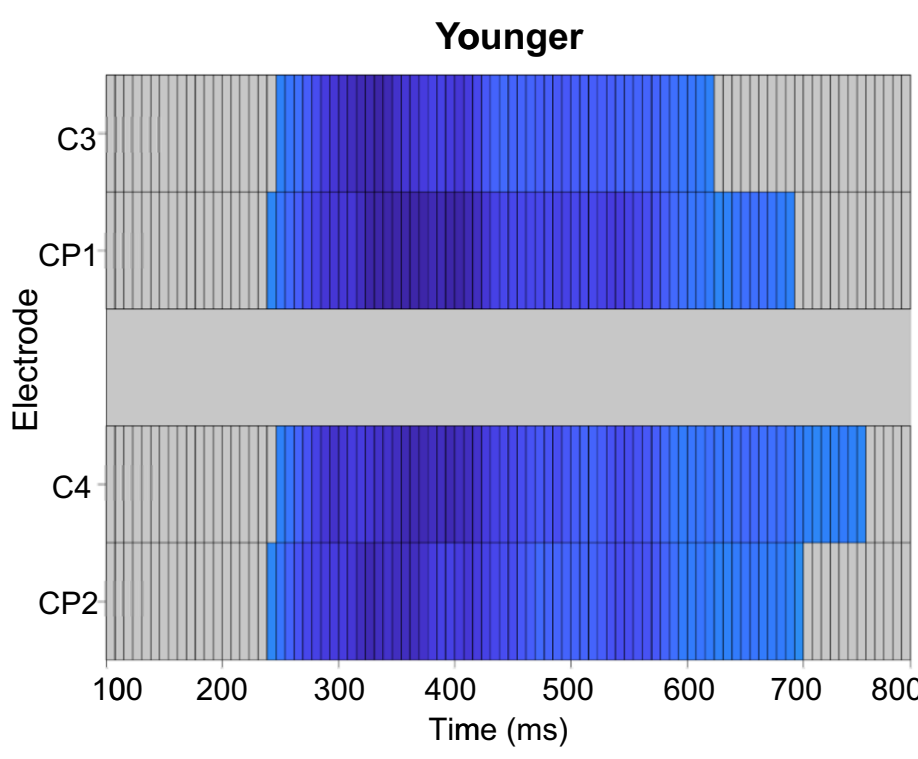

Older

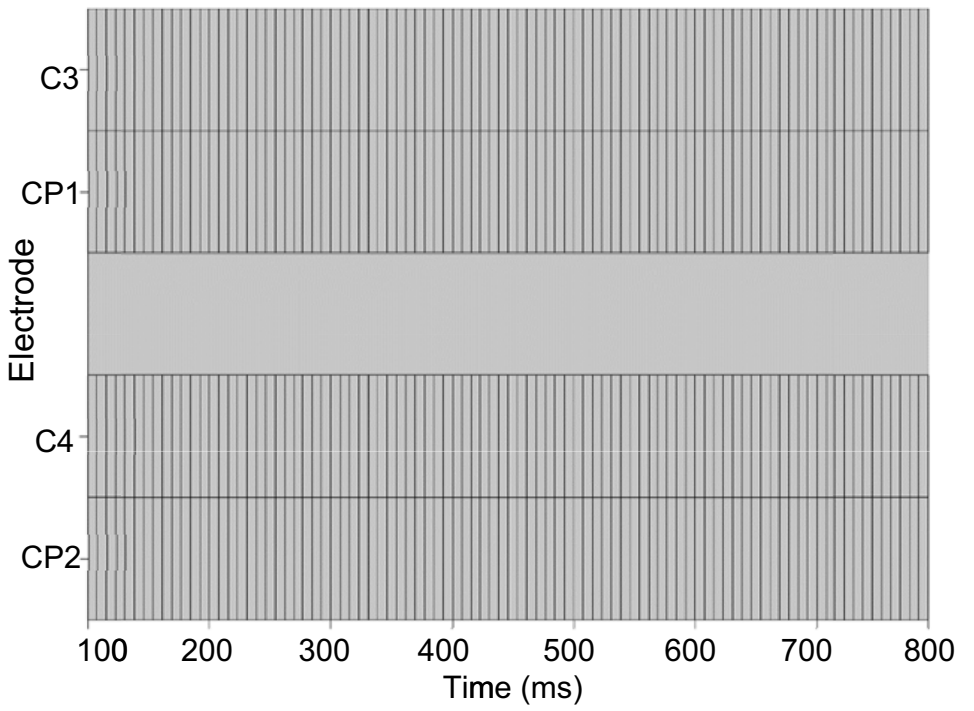

$+3.5$

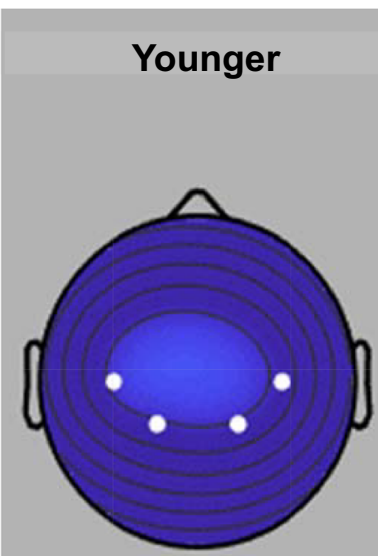

252-627 ms
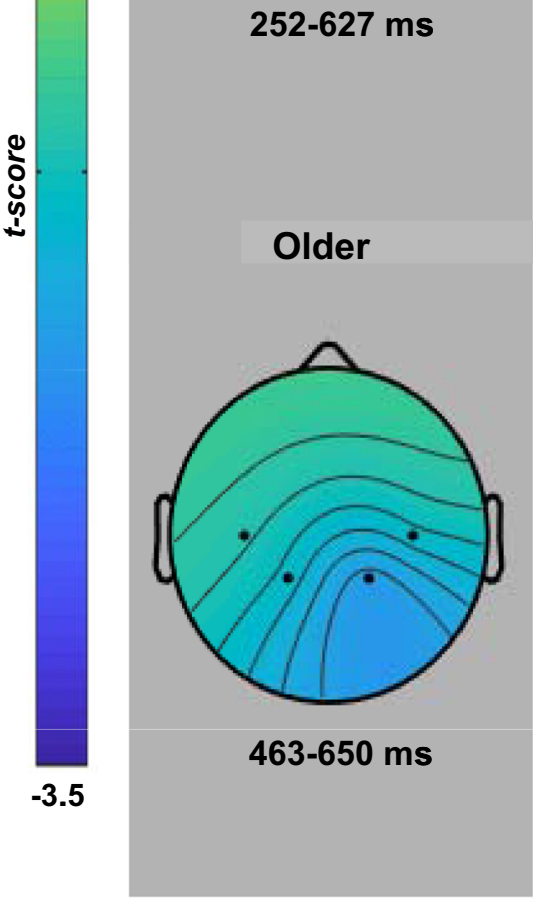

Fig. 4. Bar task results. Left-hand panel: Raster diagrams visualizing the results of cluster-based permutation analyses on ERPs for the contrast comparing emotional and neutral images. Each cell represents the result of a $t$-test on the contrast. The $\mathrm{y}$-axis indicates electrodes. The $\mathrm{x}$-axis indicates time in $13 \mathrm{~ms}$ increments. Color indicates that the test was

significant $(p<0.05)$ after correcting for multiple comparisons. Righthand panel: Topographical maps reflecting difference scores for ERPs to emotional versus neutral images. Circles represent electrode locations $(\mathrm{C} 3 / \mathrm{C} 4, \mathrm{CP} 1 / \mathrm{CP} 2)$ for the cluster analysis

Older adults The ANOVA during the 463-650 ms time window $^{7}$ did not revealed a main effect of Valence $\left[F(2,62)=0.27, p=0.76, \eta^{2}=0.01\right]$. ERPs were similar across Valence categories (Fig. 7). Because there were no effects of valence, difference scores associated with positivity, negativity, or valence were not correlated with bar task accuracy.

\footnotetext{
${ }^{7}$ Because older adults did not show any emotion-related effects in the bar task, the time window selected is the time window where we observed emotionrelated effects in the picture task.
}

Between-group analyses Figure 8 displays difference waveforms reflecting positivity (positive minus neutral), negativity (negative minus neutral), and emotional salience (average emotional minus neutral) effects in the bar task. During the window associated with the late-onset positivity component in each age group, the analyses (using a Bonferroni-corrected $\alpha$ $=0.0167$ ) revealed that ERP difference scores reflecting the positivity and emotional salience effects were reliably larger in the younger group compared to the older group [all $t s>3$, $p s<0.004$ ], with no significant age-related difference regarding the negativity effect $[t(62)=2.29, p=0.03]$. 
Younger
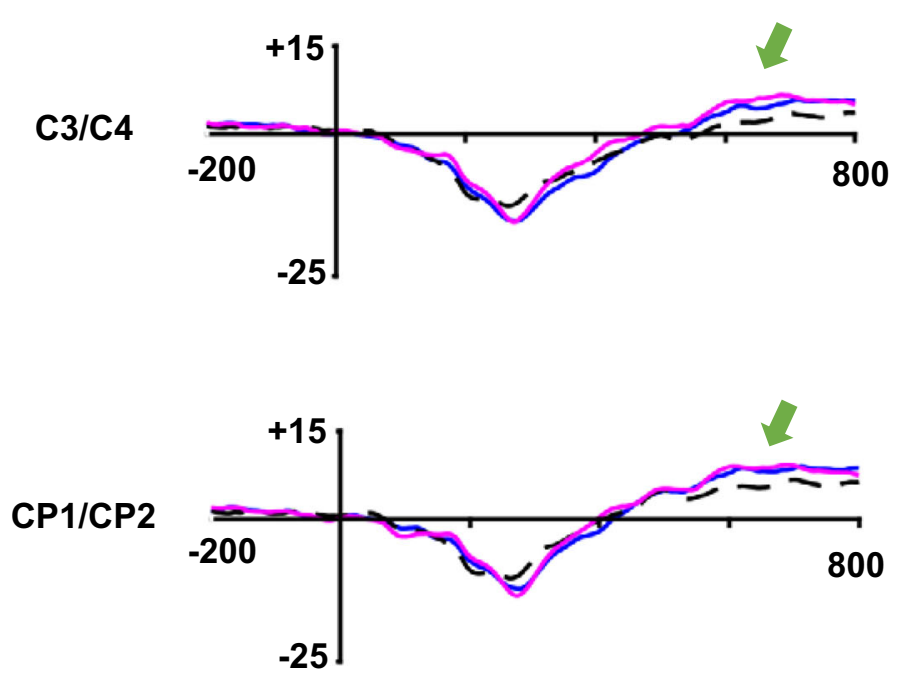

Older
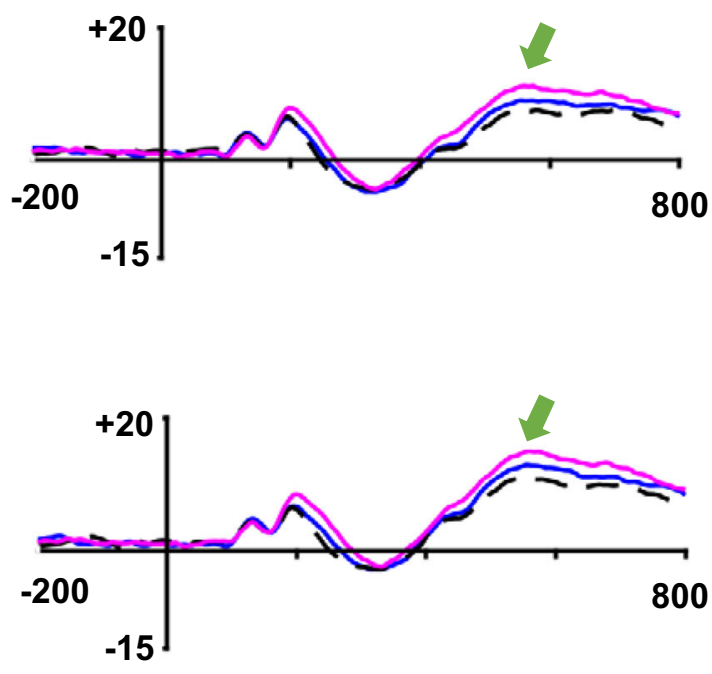

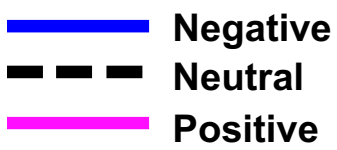

Fig. 5. ERPs elicited by negative, neutral, and positive images in the picture task by younger and older adults. Green arrows indicate the late-onset positivity effect in younger $(564-775 \mathrm{~ms})$ and older $(463-650 \mathrm{~ms})$ adults

\section{Discussion}

In the current study, we examined ERP correlates of agerelated differences in processing of task-relevant and irrelevant emotional material when performance between age groups was equated at baseline. We employed a selective attention task in which younger and older adults were presented with a picture (emotionally neutral, positive, or negative) flanked by two bars; the task was to denote whether the picture was presented in color or whether the orientation of the bars matched. There were three major findings. First, at the behavioral level, when images were task-relevant, accuracy was higher for emotional relative to neutral material regardless of age; when images were taskirrelevant, there was no interference from emotional material. Second, when images were task-relevant, ERPs provided evidence for emotion-based processing in the younger group; older adults showed more differentiated valence-based processing, reflecting a positivity effect. Third, when images were task-irrelevant, ERP patterns differed by age. Older adults showed effective inhibition of the emotional distractors (i.e., they processed emotional material at the level of neutral material); younger adults engaged in suppression (i.e., we found evidence for reduced processing of task-irrelevant emotional images relative to baseline neutral images). These results and their implications are discussed in more detail below.

\section{Behavioral results}

Although both younger and older showed higher accuracy for emotional relative to neutral images in the picture task, there was no evidence of valence-based effects (either a positivity or negativity bias) in either age group. Performance was close to ceiling, however, and this is likely to have masked potential valencebased effects. Crucially, in the bar task, neither accuracy nor response times differed as a function of emotion, suggesting that neither age group experienced intrusion from emotional material at the behavioral level. This absence of interference from taskirrelevant emotional images in the bar task seems to be in line with the load theory of attention (Lavie, 1995), which predicts that difficult tasks (like our bar task, which was engineered to yield $75 \%$ accuracy) leave no additional resources for processing task-irrelevant material. As is typical, we observed age-related slowing, reflected in longer response times in the older compared to the younger group in both picture and bar tasks.

\section{ERP Results}

Emotional processing in the picture task The picture task offers insights into the neural responses to emotional pictures that were not explicitly scrutinized for emotional content. In the younger group, the late-onset positivity, which was observed from 564 to $775 \mathrm{~ms}$, was larger for emotional relative to neutral images, suggesting that in this time window 


\section{Positivity}

$\mathrm{C} 3 / \mathrm{C} 4$

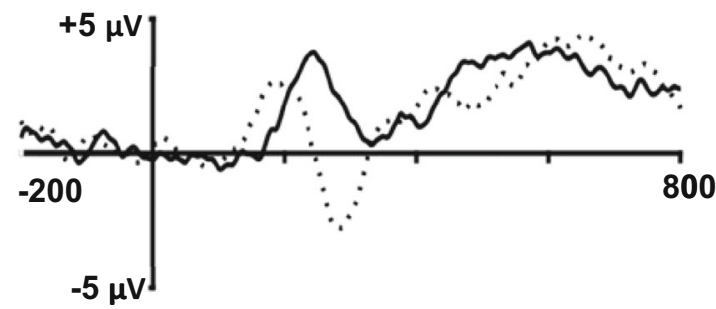

CP1/CP2

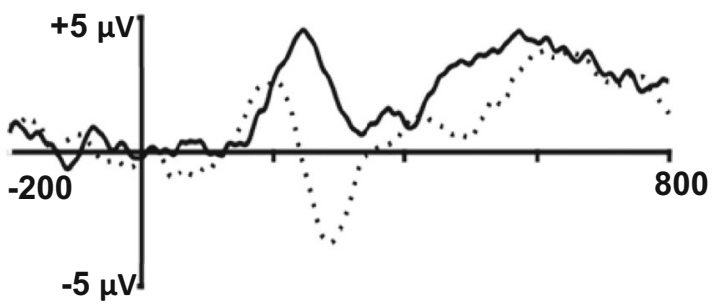

Negativity
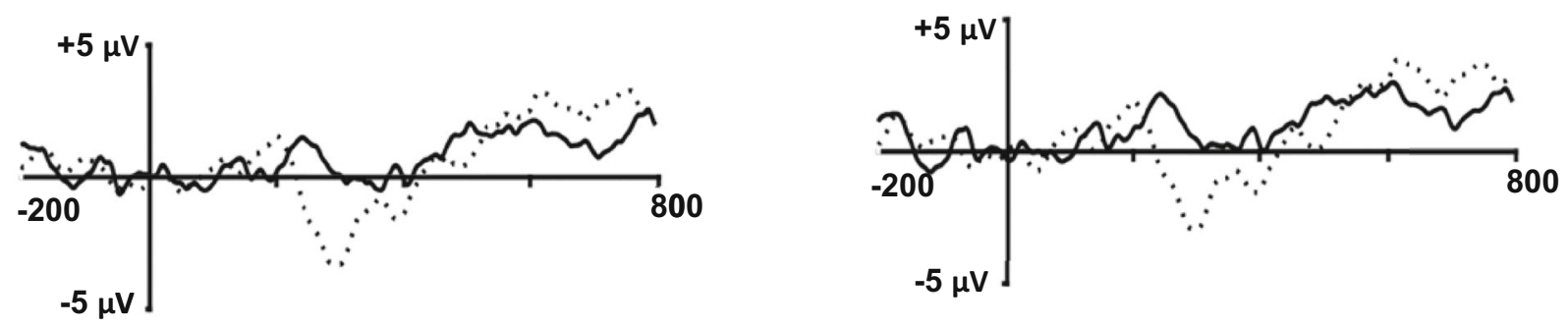

Emotional Salience
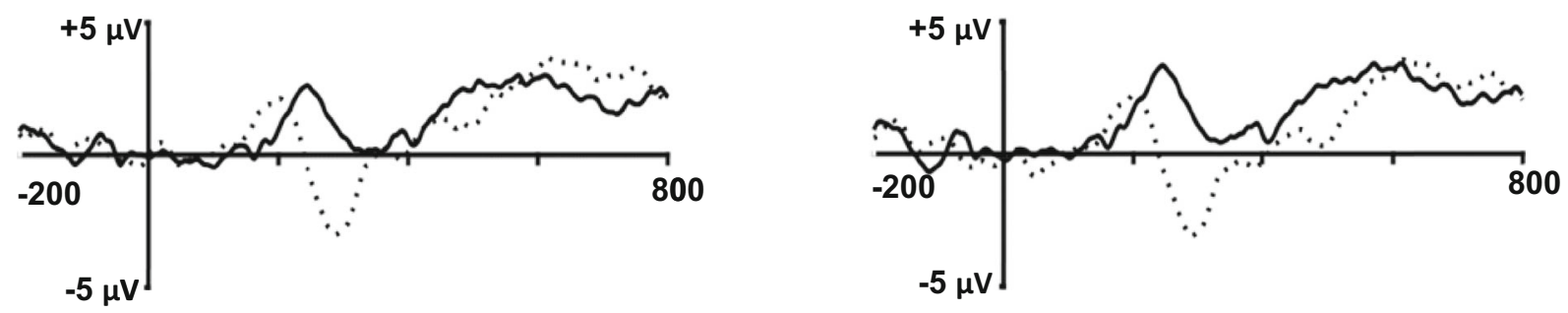

\section{......... Younger \\ Older}

Fig. 6. Difference waveforms for ERP difference scores reflecting positivity (top row), negativity (middle row), and emotional salience (bottom row) effects in the picture task at electrode sites of interest for

younger adults are sensitive to the difference in arousal value between stimuli. In the older group, the late-onset positivity, which was observed from 463 to $650 \mathrm{~ms}$, was associated with larger ERPs for positive compared with negative and neutral images, suggesting that older adults showed increased attention to motivationally salient positive material, which in turn may reflect semantic elaboration focusing on the positive content. This finding is consistent with previous ERP studies showing larger late-onset positivity for positive relative to negative images (Kisley et al., 2007; Mathieu et al., 2014). It also may imply that the older adults engaged in some form of emotion-regulation mechanism that accentuated the positive content of the pictures (Carstensen et al., 1999). younger (dotted black) and older (solid black) adults; no significant agerelated differences were found

Interestingly, although older adults showed a valence-based effect, reflecting a positivity bias, no valence-based effect reflecting negativity bias was observed in younger adults. Instead, the younger group showed an overall emotion-based effect. Although this ERP pattern is consistent with previous studies with younger adults showing an increase in magnitude of the late-onset positivity for both pleasant and unpleasant compared with neutral stimuli (Cuthbert et al., 2000; Schupp et al., 2000), it is inconsistent with some previous studies providing evidence for a negativity bias during the time window associated with the late-onset positivity (Ito et al., 1998). One possible reason for this may be that the valence effect might be moderated by arousal. Wiens and Syrjänen (2013) showed that when 


\section{Younger}
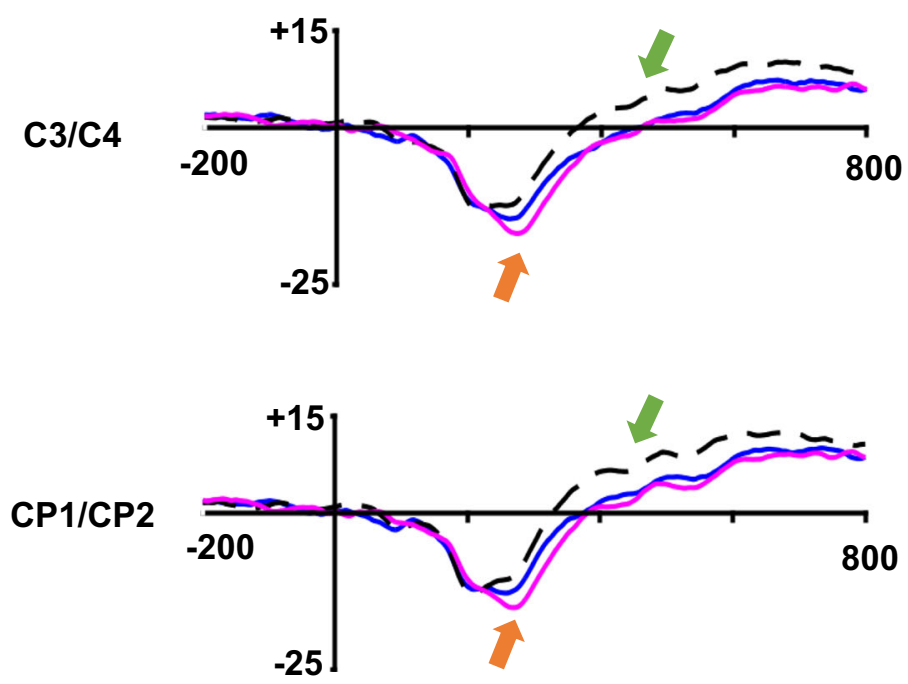

Older
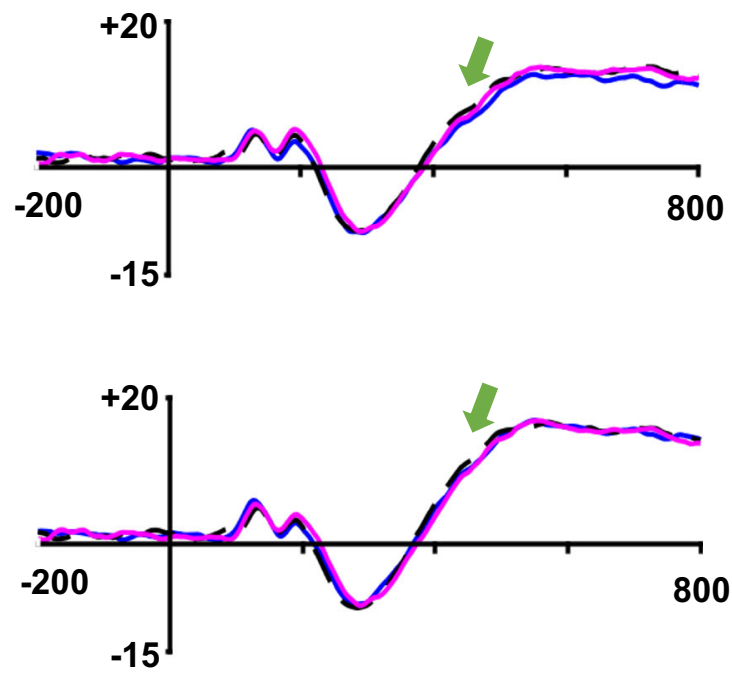

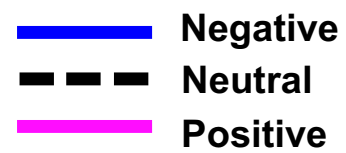

Fig. 7. ERPs elicited by negative, neutral, and positive images in the bar task by younger and older adults. Orange arrows indicate the sustained negativity effect observed in younger adults (252-400 ms); green arrows

emotional material is task-relevant and arousal level of the stimuli ranged from medium to high, ERPs reflecting the late-onset positivity were similar for pleasant and unpleasant images. In contrast, extreme stimulus arousal values produced valencebased effects on ERPs. Although our negative images were more arousing than our positive and neutral images, we did not include extremely arousing negative images, which in turn may have prevented the negativity bias to appear in the younger group.

One of the goals of the picture task was to elucidate the underlying mechanisms for the positivity effect in older adults. We indeed observed an emotional positivity effect during the time window associated with the late-onset positivity component in the older adult group. Between-age-group comparisons, however, yielded no age-related differences regarding ERPs for either negativity, positivity, or emotional salience during the late-onset positivity. Taken together, this set of results provides partial support for SST (Carstensen et al., 1999) and rules out ABM (Cacioppo et al., 2011). Specifically, older adults showed a positivity effect without an age-related decline in processing of negative material. Thus, this positivity effect appears not to be driven by an age-related neural deficit in processing negative material as suggested by ABM. However, given that the magnitude of the positivity effect is similar across age groups, SST cannot be fully supported, because it predicts a larger positivity effect in older adults relative to their younger counterparts. indicate the late-onset positivity effect in younger (401-627 ms) and older (463-650 ms) adults

Emotional processing in the bar task In the bar task, participants engaged in a cognitive task-judging whether the orientation of two slanted bars matched - while ignoring pictures (neutral, negative, or positive) projected at the center of the screen. Before the experiment, task performance was calibrated using neutral pictures as the distractors. Thus, the findings from this task shed light on the extent to which older and younger adults are capable of specifically inhibiting the emotional content of the pictures.

Older adults showed near-identical ERPs for task-irrelevant emotional and neutral images during the time window associated with late-onset positivity $(450-650 \mathrm{~ms})$, indicating that they did not process task-irrelevant emotional material over and beyond task-irrelevant neutral material in the bar task (i.e., distraction from emotionally charged and neutral material was similar). Thus, in line with the idea that at least some inhibitory deficits might be an artifact of task difficulty, the data suggest that when performance was equated across subjects in an emotionally neutral version of the task, older adults were indeed able to ignore task-irrelevant emotional material. This suggests, more generally, that when the main task is of sufficient difficulty, older adults are able to prioritize task-relevant stimuli, resulting in effective rejection of task-irrelevant material, as predicted by cognitive load theory (Lavie, 1995).

Different from older adults, ERP data from the younger group showed a pattern that reflects differential processing of 


\section{Positivity}

\section{$\mathrm{C} 3 / \mathrm{C} 4$}

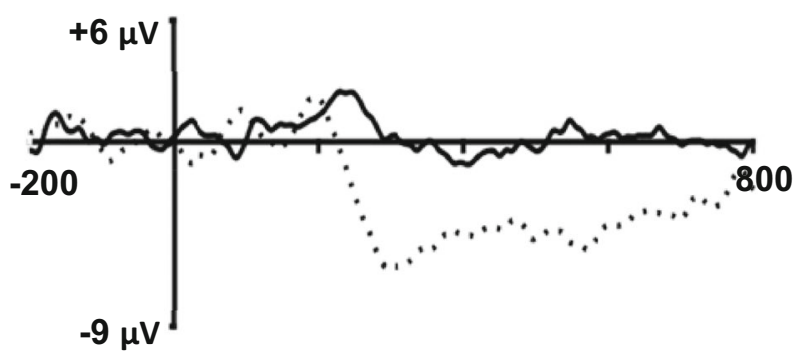

CP1/CP2

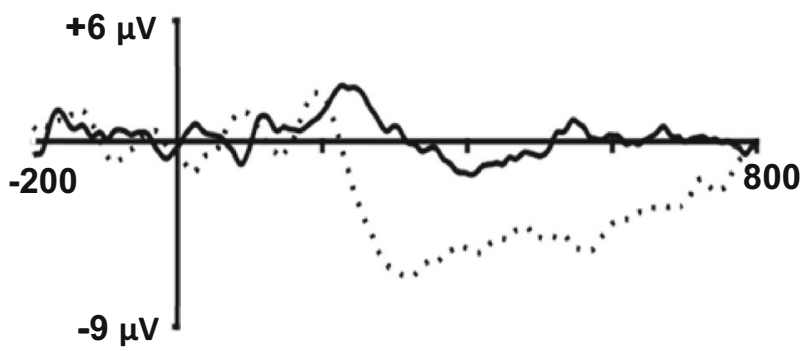

Negativity
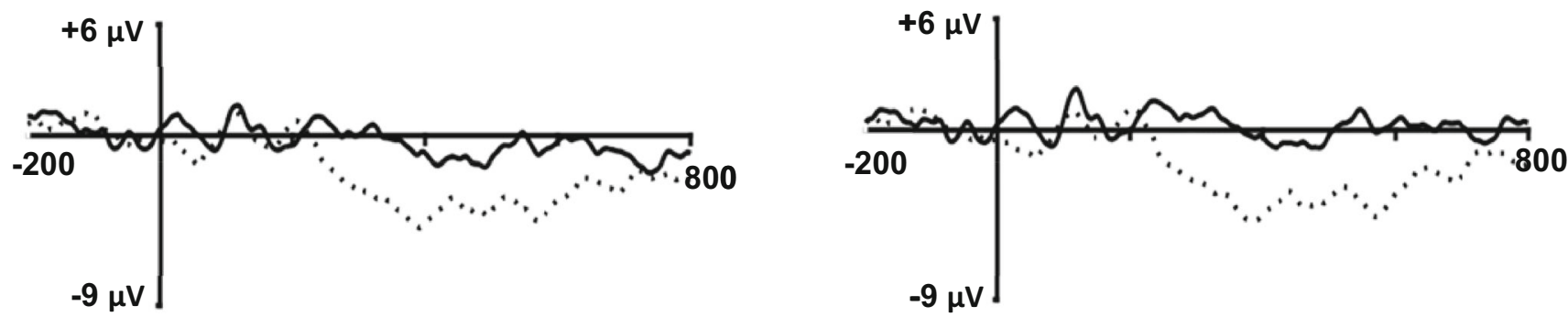

Emotional Salience
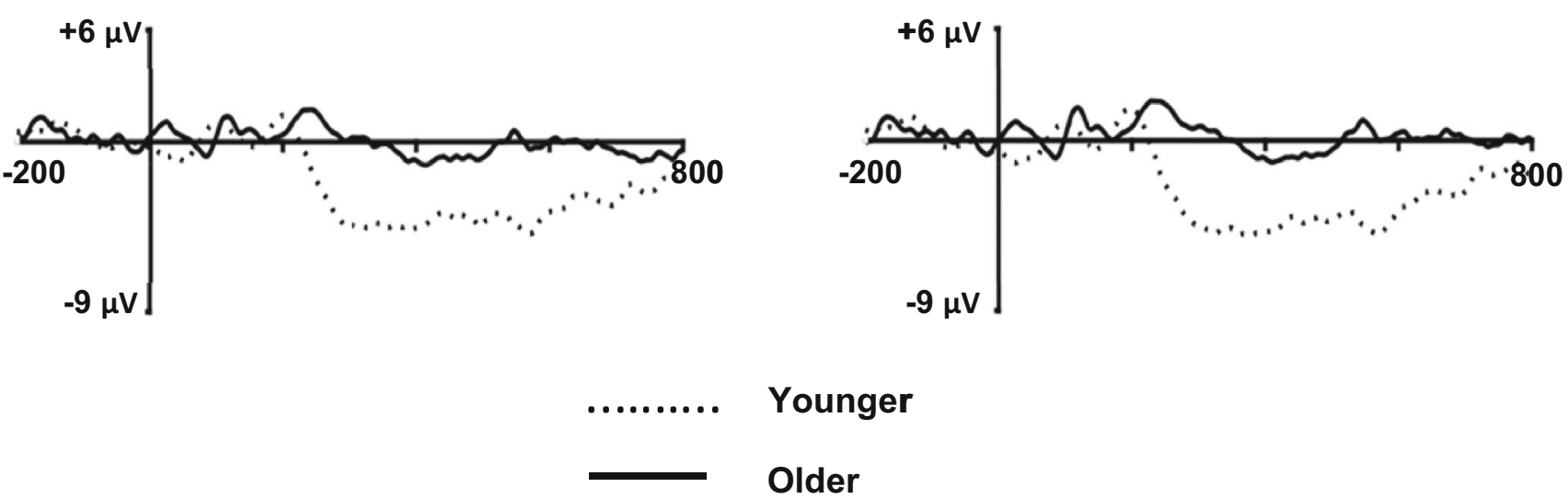

Fig. 8. Difference waveforms for ERP difference scores reflecting positivity (top row), negativity (middle row), and emotional salience (bottom row) effects in the bar task at electrode sites of interest in time windows associated with ERP effects for younger (dotted black) and older (solid black) adults

task-irrelevant material. Specifically, ERPs to task-irrelevant emotional material relative to neutral material was evident during the time window associated with the sustained negative deflection preceding the later positivity $(252-400 \mathrm{~ms})$, suggesting that younger adults did perceive and process pictures that were supposed to be ignored up to the level of extracting their emotional content. This interference from emotional material indicates that younger adults were not able or willing to inhibit emotional distractors over and above neutral distractors. During the later portion of processing (401-627 ms), however, younger adults showed larger ERPs for neutral relative to emotional images - the opposite of what would be expected for unimpeded perception of emotion and also the opposite of the pattern that was found in the picture task, where ERPs were larger for emotional than neutral images. These findings suggest that after the initial breakthrough of the emotional content of the to-be-ignored pictures earlier in the processing stream, younger adults exhibited an attentional shift that allowed them to differentiate between neutral and emotional material at the later stage of processing and suppress the emotional content. 
Importantly, between-age-group comparisons regarding the magnitude of ERPs reflecting emotional salience (i.e., ERPs to emotional versus neutral images) indicated larger suppression in the younger group compared with the older group.

The data thus reveal an interesting and unexpected agerelated difference: While older adults showed similar ERPs for task-irrelevant images regardless of valence during the time window associated with the late-onset positivity component, the younger group showed evidence for suppression of taskirrelevant emotional images (as reflected by reduced ERPs for task-irrelevant emotional images relative baseline neutral images). This suppression is effective: We found significant correlations between the degree of emotional suppression and bar task accuracy. Thus, we find an interesting dissociation, possibly hinting at strategic differences between the two age groups: Older adults achieve their $75 \%$ level of accuracy by effectively ignoring (i.e., inhibiting) the task-irrelevant emotional stimuli (as instructed); younger adults achieve this same criterion by additional processing that suppresses the emotional content of the to-be-ignored images. It is not clear if this strategic difference is an age-related compensation strategy on the part of the older adults (where the task becomes "easier to perform" if stimuli are simply ignored) or if our result is an artifact of the specific level of difficulty chosen (which might still be easily attainable by younger adults by using active suppression). This raises questions about the level of effort required in the bar task across age groups and the performance criterion employed in the current study. These questions need to be examined in future studies, possibly by varying bar task difficulty both between (by varying performance criterion) and within subjects (e.g., by varying individual threshold for angular disparity systematically, such that mismatch stimuli reflect angular difference at, below, and above threshold).

One limitation of the current study is that the participants were not screened for cognitive impairments or mood disorders using objective measures. Growing evidence shows potentially compensatory role of brain regions relevant to cognitionemotion interactions in patients with mild cognitive impairment (MCI) during working memory tasks involving emotional distractors. In particular, previous studies found an increased activation in amygdala and prefrontal regions (Berger et al., 2015), as well as anterior cingulate (Burhan et al., 2016) in MCI patients relative to healthy controls, especially at higher levels of difficulty of working memory tasks performed in the context of task-irrelevant emotional material. Although none of our participants reported conditions known to impair cognitive and affective functioning (e.g., Parkinson's or Alzheimer's disease; stroke; epilepsy; multiple sclerosis; head injury or brain damage; depression or anxiety), a more detailed neuropsychological profiling based on objective instead of self-report measures could be a fruitful avenue for future research to better delineate impact of individuals differences on neural underpinnings of cognition-emotion interactions.

\section{Conclusions}

We found that when emotional material is task-relevant younger adults only discriminate emotional content from nonemotional content as evidenced in ERPs, whereas ERPs in older adults provide evidence for a selective bias towards positive material. Although processing of negative material did not differ by age, there also was no age-related differences in the magnitude of the positivity effect. Thus, the absence of agerelated differences in processing of negative material combined with the positivity effect observed within the older group provides partial support to the claim that the wellknown age-related positivity effect arises from an increased focus on positive material as suggested by SST (Carstensen et al., 1999), rather than a reduced negativity bias in older adults as suggested by ABM (Cacioppo et al., 2011).

When emotional material is task-irrelevant, ERP amplitudes for emotional and neutral images in the older group were virtually identical, suggesting that older adults are capable of inhibiting irrelevant emotional material over and above neutral material, even if this material is presented at fixation. These results are consistent with perceptual load theory (Lavie, 1995), which argues that when the task is sufficiently demanding, individuals prioritize task-relevant stimuli, resulting in effective rejection of task-irrelevant material. Younger adults showed larger suppression of emotional distractors relative to older adults, in the absence of age-related differences at baseline. More research is needed to examine the exact mechanism behind this age-related difference in task approach.

Acknowledgements The authors thank their research assistants, Lindsey Drummond, Amelia Tan, Carrie Brooks, and Can Ozuretmen for their contribution to this study. This work was partially supported by the APA Dissertation Award awarded to Didem Pehlivanoglu. Programming scripts can be found at https://osf.io/rmfj6/. None of the experiments was preregistered.

\section{Appendix}

\section{Procedure for the Picture Rating Experiment}

Eighty-eight younger (34 females) and 90 older (52 females) adults participated in the picture rating experiment designed with Qualtrics survey software. Participants were recruited through MTurk, which is an internet-based platform that allows one to request jobs, such as survey completions, from participants seeking monetary compensation. MTurk facilitates highquality data collection from a large pool of diverse participants by allowing job requesters to reject participants' work if they do not follow instructions. For the purposes of the current rating study, I limited participation to individuals whose age ranged from 18-25 years (for younger adults) or 60-80 years (for older adults) and who were located in the United States, to 
reduce potential cultural differences in perception of images (Gruhn \& Scheibe, 2008). To get more reliable data, participation was limited to individuals who had demonstrated reliable MTurk performance in the past (HIT approval rate $>98 \%$ ) and had sufficient experience with MTurk (number of HITs approved $>1,000$ ). Additionally, self-reported colorblind subjects were excluded from the study. Participants were paid $\$ 4 /$ hour as compensation for participation. The mean age of younger adults was $22.5(S D=1.89)$; the mean age of older adults was $63.38(S D=5.32)$. Older adults $(M=15.45, S D=2.81)$ had completed more years of education than younger adults $(M=$ $14.53, S D=1.62), t(175)=2.65, p=0.009$.

For the picture rating experiment, 720 images (see pg. 15 for sources of the images and the criteria used to create the stimulus pool in the picture rating experiment) were divided into 4 groups of 180 pictures, which included an equal number of images (60) from 3 predefined valence categories (i.e., positive, negative, neutral). Each group of pictures were rated by 10 younger and 10 older adults. There were two blocks of picture rating. In one of the blocks, the subjects completed a valence rating for 180 pictures; in the other block, they completed an arousal rating for the same 180 pictures. The order of rating blocks was counterbalanced. Before starting the rating study, younger and older adults electronically signed a consent form and filled out a brief demographic information form. During the picture rating study, the subjects were first presented with a fixation cross on the screen. After presentation of the fixation cross, a picture was presented at fixation for $200 \mathrm{~ms}$ for the younger participants; this presentation time was increased to $250 \mathrm{~ms}$ for the older participants to compensate for agerelated slowing in perceptual processing (Verhaeghen, 2013). Next, participants were asked to rate the presented picture using the Self-Assessment Manikin (SAM) scale (Bradley \& Lang, 1994). The SAM is a visual analog scale portraying a series of graphical figures ranging from 1 (extremely pleasant) to 9 (extremely unpleasant) for ratings of valence, and 1 (extremely aroused) to 9 (extremely calm) for ratings of arousal. Specifically, for the valence rating, the participants were asked to decide how positive or negative an image made them feel according to the following instructions: "This scale is the happy-unhappy scale, which ranges from a smile to a frown. At one extreme of the happy vs. unhappy scale, you felt happy, pleased, satisfied, contented, and hopeful. These feelings are represented by the figure number 1 in the scale. So, if you felt completely happy while viewing the picture, you can indicate this by clicking on the figure number 1 . The other end of the scale is when you felt completely, unhappy, annoyed, unsatisfied, melancholic, despaired, bored. These feelings are represented by the figure number 9 in the scale. Thus, you can indicate feeling completely unhappy by clicking the figure number 9. If you felt completely neutral, neither happy nor $\mathrm{sad}$, indicate this by clicking on the figure 5 . The figures also allow you to describe intermediate feelings of pleasure. You can indicate intermediate feelings of pleasure by clicking on the figures number 3 and 7 . To make more finely graded judgments of pleasure or displeasure, you can click on the digits 2, 4, 6, and 8 on the scale." For the arousal rating, participants were asked to decide how calm or excited the picture made them feel, according to the following instructions: "At one extreme of the scale you felt stimulated, excited, frenzied, jittery, wide-awake, aroused. These feelings are represented by the figure number 1 in the scale. So, if you felt completely aroused while viewing the picture, you can indicate this by clicking on the figure 1. At the other end of the scale, you felt completely relaxed, calm, sluggish, dull, sleepy, unaroused. These feelings are represented by the figure number 9 in the scale. Thus, you can indicate you felt completely calm by clicking on the figure 9 . If you are not at all excited nor at all calm, click on the figure 5. Intermediate levels of excitement are represented by the figures number 3 and 7. To make a more finely tuned rating of how excited or calm you feel, you can click on the digits $2,4,6$, and 8 on the scale." Once the participant made their response, the next image was presented. After completing all ratings, the participants were asked to report whether they encountered any technical problems while completing the survey. Additionally, they were asked to report their viewing distance and screen resolution, because the viewing distance and screen resolution may influence perception of pictures. The study took approximately 1 hour for the younger adults and 1.5 hours for the older adults.

\section{References}

Anderson, A. K., \& Phelps, E. A. (2001). Lesions of the human amygdala impair enhanced perception of emotionally salient events. Nature, 411(6835), 305.

Backs, R. W., da Silva, S. P., \& Han, K. (2005). A comparison of younger and older adults' self-assessment manikin ratings of affective pictures. Experimental Aging Research, 31(4), 421-440.

Baumeister, R. F., Bratslavsky, E., Finkenauer, C., \& Vohs, K. D. (2001). Bad is stronger than good. Review of General Psychology, 5(4), 323.

Berger, C., Erbe, A. K., Ehlers, I., Marx, I., Hauenstein, K., \& Teipel, S. (2015). Effects of task-irrelevant emotional stimuli on working memory processes in mild cognitive impairment. Journal of Alzheimer's Disease, 44(2), 439-453.

Bradley, M. M., \& Lang, P. J. (1994). Measuring emotion: the selfassessment manikin and the semantic differential. Journal of Behavior Therapy and Experimental Psychiatry, 25(1), 49-59.

Brainard, D. H. (1997). The psychophysics toolbox. Spatial Vision, 10, 433-436.

Brosch, T., Sander, D., Pourtois, G., \& Scherer, K. R. (2008). Beyond fear: Rapid spatial orienting toward positive emotional stimuli. Psychological Science, 19(4), 362-370.

Bullmore, E. T., Suckling, J., Overmeyer, S., Rabe-Hesketh, S., Taylor, E., \& Brammer, M. J. (1999). Global, voxel, and cluster tests, by theory and permutation, for a difference between two groups of structural MR images of the brain. IEEE Transactions on Medical Imaging, 18(1), 32-42.

Burhan, A. M., Anazodo, U. C., Chung, J. K., Arena, A., Graff-Guerrero, A., \& Mitchell, D. G. (2016). The effect of task-irrelevant fearfulface distractor on working memory processing in mild cognitive 
impairment versus healthy controls: An exploratory fMRI study in female participants. Behavioural Neurology, 2016.

Cacioppo, J. T., Berntson, G. G., Bechara, A., Tranel, D., \& Hawkley, L. C. (2011). Could an aging brain contribute to subjective well-being? The value added by a social neuroscience perspective. Social Neuroscience: Toward Understanding the Underpinnings of the Social Mind, 249-262.

Carretié, L., Martín-Loeches, M., Hinojosa, J. A., \& Mercado, F. (2001). Emotion and attention interaction studied through event-related potentials. Journal of Cognitive Neuroscience, 13(8), 1109-1128.

Carstensen, L. L., Isaacowitz, D. M., \& Charles, S. T. (1999). Taking time seriously: A theory of socioemotional selectivity. American Psychologist, 54(3), 165.

Codispoti, M., De Cesarei, A., \& Ferrari, V. (2012). The influence of color on emotional perception of natural scenes. Psychophysiology, 49(1), 11-16.

Cuthbert, B. N., Schupp, H. T., Bradley, M. M., Birbaumer, N., \& Lang, P. J. (2000). Brain potentials in affective picture processing: covariation with autonomic arousal and affective report. Biological Psychology, 52(2), 95-111.

Dan-Glauser, E. S., \& Scherer, K. R. (2011). The Geneva affective picture database (GAPED): a new 730-picture database focusing on valence and normative significance. Behavior Research Methods, 43(2), 468.

De Cesarei, A., Codispoti, M., \& Schupp, H. T. (2009). Peripheral vision and preferential emotion processing. Neuroreport, 20(16), 1439-1443.

Delorme, A., \& Makeig, S. (2004). EEGLAB: an open source toolbox for analysis of single-trial EEG dynamics including independent component analysis. Journal of Neuroscience Methods, 134(1), 9-21.

Donderi, D. C. (2006). Visual complexity: a review. Psychological Bulletin, 132(1), 73.

Eimer, M., Holmes, A., \& McGlone, F. P. (2003). The role of spatial attention in the processing of facial expression: an ERP study of rapid brain responses to six basic emotions. Cognitive, Affective, \& Behavioral Neuroscience, 3(2), 97-110.

Erthal, F. S., De Oliveira, L., Mocaiber, I., Pereira, M. G., MachadoPinheiro, W., Volchan, E., \& Pessoa, L. (2005). Load-dependent modulation of affective picture processing. Cognitive, Affective, \& Behavioral Neuroscience, 5(4), 388-395.

Fabiani, M. (2012). It was the best of times, it was the worst of times: A psychophysiologist's view of cognitive aging. Psychophysiology, 49(3), 283-304.

Foti, D., Hajcak, G., \& Dien, J. (2009). Differentiating neural responses to emotional pictures: evidence from temporal-spatial PCA. Psychophysiology, 46(3), 521-530.

Gazzaley, A., Clapp, W., Kelley, J., McEvoy, K., Knight, R. T., \& D'Esposito, M. (2008). Age-related top-down suppression deficit in the early stages of cortical visual memory processing. Proceedings of the National Academy of Sciences, 105(35), 13122-13126.

Gazzaley, A., Cooney, J. W., McEvoy, K., Knight, R. T., \& D'esposito, M. (2005). Top-down enhancement and suppression of the magnitude and speed of neural activity. Journal of Cognitive Neuroscience, 17(3), 507-517.

Groppe, D. M., Urbach, T. P., \& Kutas, M. (2011). Mass univariate analysis of event-related brain potentials/fields II: Simulation studies. Psychophysiology, 48(12), 1726-1737.

Grühn, D., \& Scheibe, S. (2008). Age-related differences in valence and arousal ratings of pictures from the International Affective Picture System (IAPS): Do ratings become more extreme with age?. Behavior Research Methods, 40(2), 512-521

Hajcak, G., Dunning, J. P., \& Foti, D. (2009). Motivated and controlled attention to emotion: time-course of the late positive potential. Clinical Neurophysiology, 120(3), 505-510.

Hasher, L., Stoltzfus, E. R., Zacks, R. T., \& Rypma, B. (1991). Age and inhibition. Journal of Experimental Psychology: Learning, Memory, and Cognition, 17(1), 163.
Hasher, L., \& Zacks, R. T. (1988). Working memory, comprehension, and aging: A review and a new view. Psychology of Learning and Motivation, 22, 193-225.

Holmes, A., Kiss, M., \& Eimer, M. (2006). Attention modulates the processing of emotional expression triggered by foveal faces. Neuroscience Letters, 394(1), 48-52.

Holmes, A., Vuilleumier, P., \& Eimer, M. (2003). The processing of emotional facial expression is gated by spatial attention: evidence from event-related brain potentials. Cognitive Brain Research, 16(2), 174-184.

Huang, Y. X., \& Luo, Y. J. (2006). Temporal course of emotional negativity bias: an ERP study. Neuroscience Letters, 398(1-2), 91-96.

Ito, T. A., Larsen, J. T., Smith, N. K., \& Cacioppo, J. T. (1998). Negative information weighs more heavily on the brain: the negativity bias in evaluative categorizations. Journal of Personality and Social Psychology, 75(4), 887.

Kisley, M. A., Wood, S., \& Burrows, C. L. (2007). Looking at the sunny side of life: Age-related change in an event-related potential measure of the negativity bias. Psychological Science, 18(9), 838-843.

Kurdi, B., Lozano, S., \& Banaji, M. R. (2017). Introducing the open affective standardized image set (OASIS). Behavior Research Methods, 49(2), 457-470.

Lang, P. J., Bradley, M. M., \& Cuthbert, B. N. (1999). International affective picture system (IAPS): Technical manual and affective ratings. Gainesville, FL: The Center for Research in Psychophysiology, University of Florida.

Lavie, N. (1995). Perceptual load as a necessary condition for selective attention. Journal of Experimental Psychology: Human Perception and Performance, 21(3), 451.

Lopez-Calderon, J., \& Luck, S. J. (2014). ERPLAB: an open-source toolbox for the analysis of event-related potentials. Frontiers in Human Neuroscience, 8, 213.

Lustig, C., Hasher, L., \& Zacks, R. T. (2007). Inhibitory deficit theory: Recent developments in a "new view". Inhibition in Cognition, 17, 145-162.

MacNamara, A., \& Hajcak, G. (2009). Anxiety and spatial attention moderate the electrocortical response to aversive pictures. Neuropsychologia, 47(13), 2975-2980.

Manly, B. F. J. (1997). Randomization, bootstrap, and Monte Carlo methods in biology (2nd ed.). London: Chapman \& Hall.

Marchewka, A., Żurawski, Ł., Jednoróg, K., \& Grabowska, A. (2014). The Nencki Affective Picture System (NAPS): Introduction to a novel, standardized, wide-range, high-quality, realistic picture database. Behavior Research Methods, 46(2), 596-610.

Maris, E., \& Oostenveld, R. (2007). Nonparametric statistical testing of EEG- and MEG-data. Journal of Neuroscience Methods, 164(1), 177-190.

Mathieu, N. G., Gentaz, E., Harquel, S., Vercueil, L., Chauvin, A., Bonnet, S., \& Campagne, A. (2014). Brain processing of emotional scenes in aging: effect of arousal and affective context. PloS ONE, 9(6), e99523.

Nuwer, M. R., Comi, G., Emerson, R., Fuglsang-Frederiksen, A., Guérit, J. M., Hinrichs, H., ... Rappelsburger, P. (1998). IFCN standards for digital recording of clinical EEG. Clinical Neurophysiology, 106(3), 259-261.

Öhman, A., Flykt, A., \& Esteves, F. (2001). Emotion drives attention: detecting the snake in the grass. Journal of Experimental Psychology: General, 130(3), 466.

Padmala, S., \& Pessoa, L. (2014). Motivation versus aversive processing during perception. Emotion, 14(3), 450.

Pelli, D. G. (1997). The VideoToolbox software for visual psychophysics: Transforming numbers into movies. Spatial Vision, 10(4), 437-442.

Pessoa, L., Kastner, S., \& Ungerleider, L. G. (2002). Attentional control of the processing of neutral and emotional stimuli. Cognitive Brain Research, 15(1), 31-45. 
Reed, A. E., Chan, L., \& Mikels, J. A. (2014). Meta-analysis of the agerelated positivity effect: age differences in preferences for positive over negative information. Psychology and Aging, 29(1), 1.

Sand, A., \& Wiens, S. (2011). Processing of unattended, simple negative pictures resists perceptual load. Neuroreport, 22(7), 348-352.

Schupp, H. T., Cuthbert, B. N., Bradley, M. M., Cacioppo, J. T., Ito, T., \& Lang, P. J. (2000). Affective picture processing: the late positive potential is modulated by motivational relevance. Psychophysiology, 37(2), 257-261.

Schupp, H. T., Flaisch, T., Stockburger, J., \& Junghöfer, M. (2006). Emotion and attention: event-related brain potential studies. Progress in Brain Research, 156, 31-51.

Shipley WC. Institute of Living Scale. Los Angeles: Western Psychological Services; 1946.

Smith, A. (1973). The Symbol Digit Modalities Test. Los Angeles: Western Psychological Services.

Verhaeghen, P. (2011). Aging and executive control: Reports of a demise greatly exaggerated. Current Directions in Psychological Science, 20(3), 174-180.

Verhaeghen, P. (2013). The elements of cognitive aging: Meta-analyses of age-related differences in processing speed and their consequences. Oxford University Press.

Verhaeghen, P., Geigerman, S., Yang, H., Montoya, A. C., \& Rahnev, D. (2019). Resolving age-related differences in working memory:
Equating perception and attention makes older adults remember as well as younger adults. Experimental Aging Research, 1-15.

Watson, A. B., \& Pelli, D. G. (1983). QUEST: A Bayesian adaptive psychometric method. Perception \& Psychophysics, 33(2), 113-120.

Weinberg, A., \& Hajcak, G. (2010). Beyond good and evil: The timecourse of neural activity elicited by specific picture content. Emotion, 10(6), 767.

Weinberg, A., \& Hajcak, G. (2011). The late positive potential predicts subsequent interference with target processing. Journal of Cognitive Neuroscience, 23(10), 2994-3007.

Wiens, S., Sand, A., \& Olofsson, J. K. (2011b). Nonemotional features suppress early and enhance late emotional electrocortical responses to negative pictures. Biological Psychology, 86(1), 83-89.

Wiens, S., Sand, A., Norberg, J., \& Andersson, P. (2011a). Emotional event-related potentials are reduced if negative pictures presented at fixation are unattended. Neuroscience Letters, 495(3), 178-182.

Wiens, S., \& Syrjänen, E. (2013). Directed attention reduces processing of emotional distracters irrespective of valence and arousal level.

Publisher's note Springer Nature remains neutral with regard to jurisdictional claims in published maps and institutional affiliations. 